\title{
DESIGN, FABRICATION, AND COMPARATIVE STUDY OF ELECTRONICALLY TUNABLE ACTIVE FILTERS
}

\author{
UMESH KUMAR* \\ Senior Member, IEEE and Fellow, IETE Department of Electrical Engg., \\ IIT New Delhi Pin-110076 India. \\ P. BHUSHAN MITAL, \\ Fellow, IETE. \\ Department of Electronics \& Communication Engg. \\ C.R. State College of Engineering, Murthal (Sonepat) India Pin-131039.
}

The Kerwin-Huelsman Newcomb (KHN) biquad is the natural choice for use as a 'universal' filter building block out of the several existing 3-amplifier biquadratic filter sections based on the two integrator loop or state variable realization of 2 nd order systems. A modified version of the KHN biquad, which is electronically tunable almost linearly over a decade range of center frequency, is presented. For electronic tunability, instead of the direct use of a FET as a voltage variable resistance to control the time constants of the integrators, a novel approach of using 'voltage-controlled variablegain amplifiers at the inputs of both the integrators and one immediately after the input signal has been suggested. The circuit is suitable for use as a tunable universal filtering module and can be easily digitally programmed.

Comparative treatment of general tuning methods of active filters, and some methods of electronic tuning of active filters are discussed. A brief description of tuning algorithms of active filters is also given.

\section{INTRODUCTION}

The general area of electrical engineering involving selective processing of signal information, commonly referred to as filtering, is a field that has broadened tremendously in scope in the past few decades. Some of the advances have been spurred by technological developments such as the tremendous increase in production and utilization of integrated circuits. Other advances have been brought about by the theoretical developments made by researchers in well-established disciplines. Taken as a whole, these advances have tremendously broadened the scope of filter theory, which, not too many years ago, was primarily concerned only with methods for interconnecting lumped resistors, capacitors, and inductors. Thus, a modern view of filter theory must necessarily include current results concerning the effects of active, distributed, and non-linear phenomena, as well as the more familiar passive, lumped, and linear situations. In addition, modern filter theory must not only consider the processing of signals by continuous filters, but also by digital or sampling techniques.

Contact Address: Dr. Umesh Kumar Assistant Professor, Department of Electrical Engineering, I.I.T., Hauz Khas, New Delhi-110016, INDIA Phone: + (91)-(11)-3263535 (Residence + (91)-(11)666979/Extn.2218 (office) FAX: + (91)-6862037 (Office) 
Filtering is a process by which the frequency spectrum of a signal can be modified, reshaped, or manipulated according to some desired specifications. It may entail amplifying or attenuating a range of frequency components, rejecting or isolating one specified frequency component, etc. The uses of filtering are manifold, e.g., to eliminate signal contamination such as noise, to remove signal distortion brought about by an imperfect transmission channel or by inaccuracies in measurement, to separate two or more distinct signals that were purposely mixed in order to maximize channel utilization, to resolve signals into their frequency components, to demodulate signals, to convert discrete-time signals into continuous time signals, and to bandlimit signals.

Filters with single pass bands are typically classified as lowpass, highpass, and bandpass, depending on the bands of frequencies that are passed. For example, the pass band of the bandpass filter illustrated in Fig. 1 extends from the frequency $\omega_{1}$ to $\omega_{2}$. There are other filter types such as allpass, band-reject, or notch filters. Another classification of filters is based on the network-theoretical concepts by which they were designed. It comprises image-parameter filters and insertion-loss filters.

An image-parameter filter consists of a cascade of two-port sections whose image impedances are matched at their junctions. If the filter is also matched at the end terminals, the 'image attenuation' would be zero in the passband. However, since the image impedance is frequency dependent and the terminations are usually resistive, the filter is not matched at its terminals at all frequencies. A non-zero attenuation in the pass band is the result.
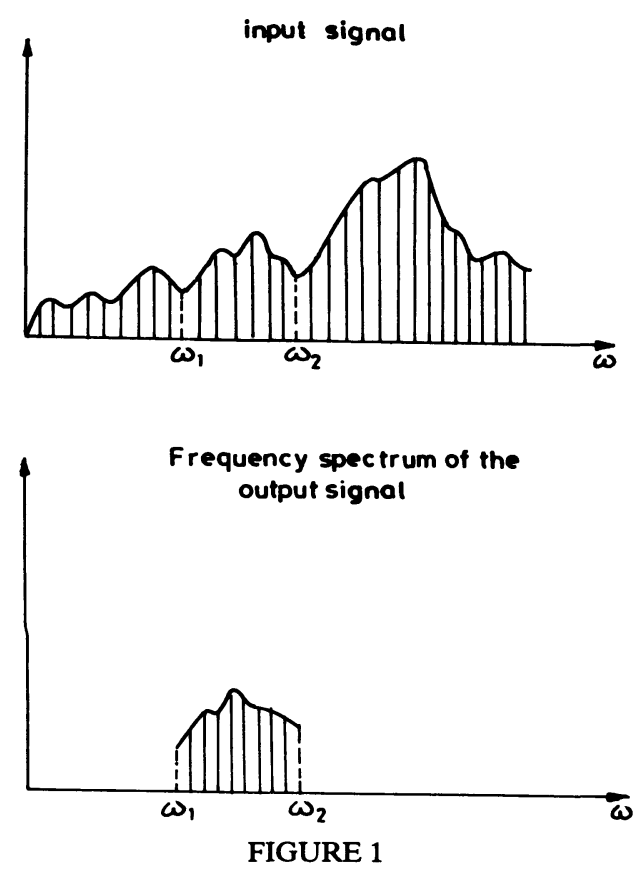
An alternative method of filter design is based on the insertion loss. Fig. 2 shows a typical requirement for a bandpass filter. The regions of high attenuation are called stop bands and the regions of low attenuation, pass bands. The nebulous region between a pass band and a stop band is termed a transition region. The insertion loss is required to remain below a certain pass band maximum Amax, measured in decibels or nepers, in the range of frequencies from $\omega_{B 1}$ to $\omega_{B 2}$, and to remain above a certain stop band minimum Amin, for frequencies lower than $\omega_{s 1}$ and above $\omega_{s 2}$. The width of the intervals $\omega_{B 1}-\omega_{S 2}$ and $\omega_{S 2}-\omega_{B 2}$ is a measure of the required frequency selectivity. The incidental power dissipation of the filter components causes the actual loss curve to depart considerably from the theoretical, especially near the edge of the band and in the vicinity of the infinite loss points. One feature of insertion-loss theory is that this non-ideal component behavior is compensated for by a predistortion technique which, however, introduces a flat loss in the pass band. The structure of the resulting insertion-loss filter is often the same as that of the equivalent image-parameter filter. For the same number of sections, the insertion-loss filter gives a better filter performance. This improvement is obtained at the cost of much greater computational effort, which was a significant deterrent before the present-day proliferation of computers. With today's vastly expanded computational capabilities, insertion-loss filter design has been made readily accessible in the form of filter tables such as those of Zverev.

Filters can be constructed using only passive components, i.e., resistors $(R)$, capacitors $(C)$, and inductors $(L)$. The passive filters are devices that take an input signal $X(t)$ and shape it to a desired output waveform $Y(t)$. A passive filter is one that does not require a power supply. Thus, a gyrator is theoretically passive, yet it will not be considered as a passive circuit element because it requires the use of a power supply. In spite of the extensive resources that have gone into the perfection of LC filter theory technology, and manufacture, there is wide spread tendency to eliminate LC filters from modern electronic equipment, because integrated circuits have completely changed conventional systems and the performance criteria previously accepted in electronic designs. Thus, LC filters, like all other circuit types

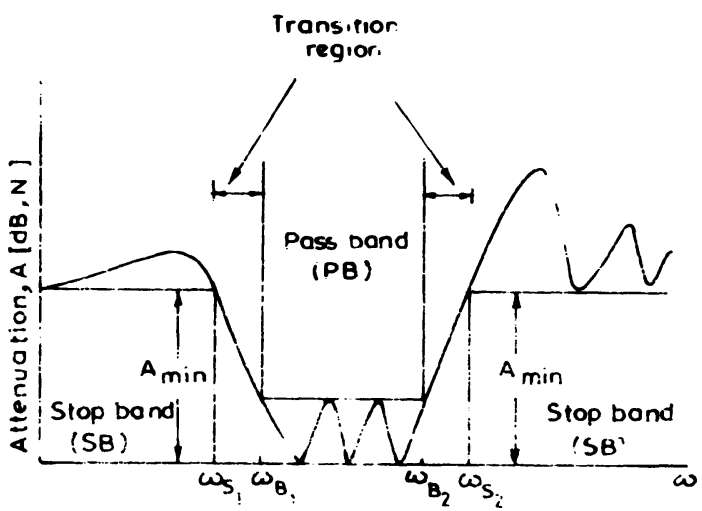

FIGURE 2 
that do not fit into the microminiaturization trend, are rapidly being replaced by filter types that do. Foremost among these are active filters that combine resistors, capacitors, and gain devices into active networks achieving filtering performance that is comparable or superior to that of their LC counterparts. Due to its excellent performance, reasonable price, and ready availability, the operational amplifier is invariably used as the active gain device.

The passive filters are mostly used at high frequencies of the order of $\mathrm{mHz}$. Especially at low frequencies (below $100 \mathrm{kHz}$ ), active filters have been offering competition to the passive filters. Active filters require a power supply. As the frequency range decreases, the size of the inductors increases. Not only do they become bulky, they also are of poorer quality - they have more loss. Active filters offer the possibility of replacing the bulky, poor quality inductor with a small integrable device. As the frequency range increases, inductors become smaller, have higher quality, and are less expensive, so that the competition they offer to active filters becomes greater. Also, most active filters do not perform well at high frequencies. Thus for economical and technological reasons, in industry active filters will be limited to frequencies below $100 \mathrm{kHz}$ for some time to come. This is not meant to indicate that no research is being performed at higher frequencies, but instead is meant as a realistic appraisal of the competition-the passive filters.

Practical active filters are often realized in hybrid form, i.e., they use thin-film circuitry to realize resistors and capacitors while they use ICs to realize the active device. In modern thin-film technology, it is possible to have resistors and capacitors that compensate each other's temperature coefficient. Also, because the resistors (and capacitors) can be on the same substrate, they tend to track. That is, if one resistor increases by $1 \%$, then all resistors tend to increase by $1 \%$ because they have been made by the same manufacturing process.

One of the original ways of realizing active filters is the negative impedance converter (NIC). This device has attracted much attention in theoretical papers, but in practice it is a poor solution to filtering problems. Gyrators are more practical than NIC's, in fact they are superior in many aspects. However, in any decision has to the type of the active device to be used in an active filter, the gyrator has a tough foe, the op-amp.

NEGATIVE IMPEDANCE CONVERTER (NIC) ACTIVE FILTER:

They are of two types

1. Current NIC

2. Voltage NIC

Both circuits are two-ports. The most important property of these two-ports is that they can be used to produce negative impedances.

In a Current NIC, $V_{1}=V_{2}, K I_{1}=I_{2}$

In a Voltage NIC, $V_{1}=-K V_{2}, I_{1}=-I_{2}$ 


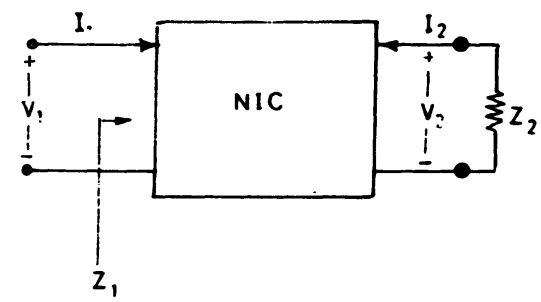

FIGURE 3

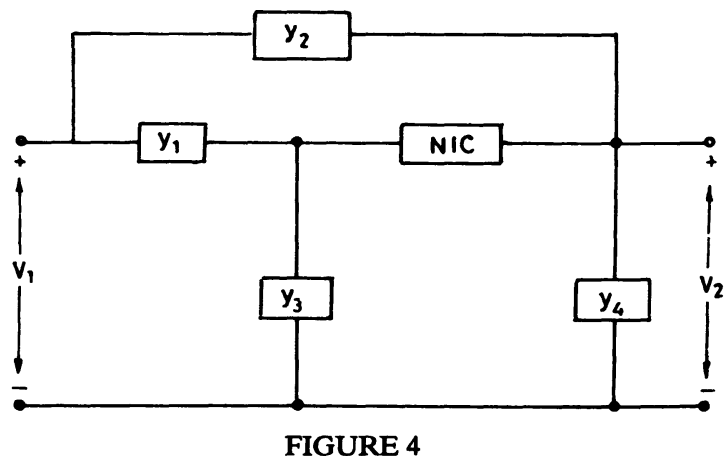

As illustrated in Fig. 3, if the $O / P$ is terminated in an impedance $Z_{2}$, then the $I / P$ impedance is $Z_{1}=-K Z_{2}$ NICs can be used to make $R C$ circuits behave like RL circuits. An example of this is shown in Fig. 4. The admittances $Y_{1}, Y_{2}, Y_{3}, Y_{4}$ are assumed to be made up of resistors and capacitors. The transfer function for the above filter configuration for a current NIC is

$\left.\frac{V_{2}}{V_{1}}\right|_{2}=0=\frac{Y_{2}-K Y_{1}}{Y_{2}+Y_{4}-K\left(Y_{1}+Y_{3}\right)}$

The disadvantages of a NIC active filter are

1. It is sensitive to component variations

2. While cascading, it needs buffer stages.

\section{GYRATOR ACTIVE FILTERS:}

A gyrator is any two-port that satisfies the matrix equation

$$
\left[\begin{array}{l}
V_{1} \\
V_{2}
\end{array}\right]=\left[\begin{array}{ll}
0 & -R_{1} \\
R_{2} & 0
\end{array}\right]\left[\begin{array}{l}
I_{1} \\
I_{2}
\end{array}\right]
$$

Where the constants $R_{1}$ and $R_{2}$ are $+V_{e}$ real numbers.

$Z_{12} \neq Z_{21} \Rightarrow$ it is non-reciprocal two-port 


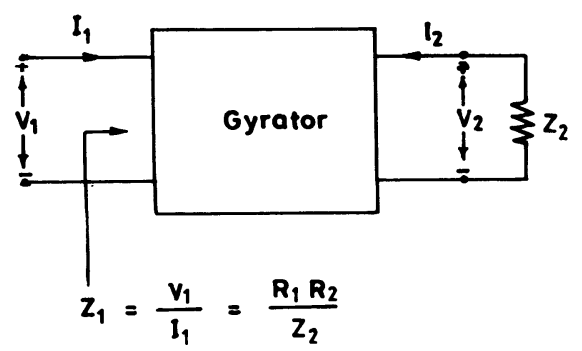

FIGURE 5

The $I / P$ and $O / P$ powers of the above gyrator are

$P_{1}=V_{1} I_{1^{*}}=-R_{1} I_{2} I_{1^{*}}$

$P_{2}=V_{2} I_{2^{*}}=R_{2} I_{1} I_{2^{*}}$

If $R_{1}=R_{2}$, the gyrator dissipates no real power and is said to be a passive device. Practical gyrators comprise many transistors and dissipate power internally. It is just at the two ports of the gyrator that the sum of the real power is zero. The most important property of the gyrator is that it can be used to invert impedances.

The fact that gyrator can be used as an impedance inverter implies that if the $O / P$ is terminated in a capacitor, then the $I / P$ impedance is that of anductor. Insertion loss filters are very insensitive to element variation in their pass band. If inductors are replaced with a gyrator-capacitor combination, the resulting active filter is insensitive, which is in sharp constrast to the highly sensitive NIC filters.

Active filters can also be constructed with gyrators by using the RC-RL partitioning method. This is somewhat analogos to the NIC synthesis procedure. The NIC synthesis procedure was based on writing a transfer function in terms of the difference of two RC impedances. The NIC was then used to produce the -ve of an RC impedance. In the RC-RL partitioning method, the transfer function is written in terms of the sum of an RC and RL impedance. The gyrator is used to produce the RL impedance from an RCimpedance. Because the RC-RL partitioning method is based on producing the sum of two terms, it is less sensitive than the NIC procedure that produces the difference of two terms.

Gyrators have not yet been mass produced and are still expensive. Besides being less expensive, op-amp active filters have other advantages: They can readily be cascaded without buffering, and they can easily provide gain at the same time they provide filtering. In special applications (such as when one may want a bilateral filter), the gyrator may prove superior.

The term 'active filters' comprises a host of different circuit concepts and design methods, the most important of which can be grouped into the following three categories.

\section{CASCADE FILTER DESIGN:}

This denotes isolated second-order filter sections (often referred to as biquadratic circuits or 'biquads') connected in cascade to realize the required higher-order 
transfer functions. The individual building blocks may be of second or third order and may comprise one or more op-amps.

\section{LC FILTER SIMULATION:}

An LC filter structure is the starting point. This is then realized either by simulating each inductor by a gyrator-capacitor combination or by transforming the initial filter structure such that it can be realized with general impedance converters (GICs), e.g., frequency dependent negative resistances (FDNRs). Both gyrators and GICs are realized with op-amps.

\section{COUPLED FILTERS:}

Here, the starting point is generally a cascade of first or second order active filters (preferably all purpose building blocks), which are then coupled by additional negative feed back loops. The additional coupling is introduced in order to obtain the same stability with the resulting active filter cascade as is obtainable with simulated LC filter structures.

\section{TUNING ACTIVE FILTERS:}

As active filters are increasingly being developed for modern communication systems, so the question of how to tune them to specifications most efficiently, and at minimum cost, is becoming every more important. One of the practical problems in manufacturing such filters is that of achieving the desired response in view of random component variations and parasitic effects. The tuning problem is concerned with devising algorithms to correct for this by adjusting the components after production. Complicating the issue are the complex non-linear equations involved; the need for efficient, computer implementable routines; and the restriction that only a subset of the components are adjustable. In particular, it is usually only possible to trim the resistors and only in an irreversible, increasing manner.

In practice two basically different tuning methods can be distinguished, namely, functional and deterministic tuning.

Functional tuning implies tuning the critical parameters of a network while it is functional, i.e., in operation. Because the network is assembled as if for operation in the final system, any parasitics built into the network are automatically taken into account and 'tuned out' during the tuning process. Functional tuning is generally iterative, particularly if the tuning steps are interactive (see Fig. 6). The number of iterations increases with the degree of tuning accuracy required. The larger the number of iterative tuning steps, the more time consuming, and, therefore, the more costly the tuning process will be. Functional tuning is generally preferable for laboratory purposes and when production quantities are moderate or low.

Deterministic tuning implies tuning- or trimming to value-individual components of a network as predicted by a combination of comprehensive network equations (in which parasitic effects are taken into account) and by component measurements (see Fig. 7). The solutions of the equations (generally obtained by an on-line computation facility) provide the values of the components to be tuned. 


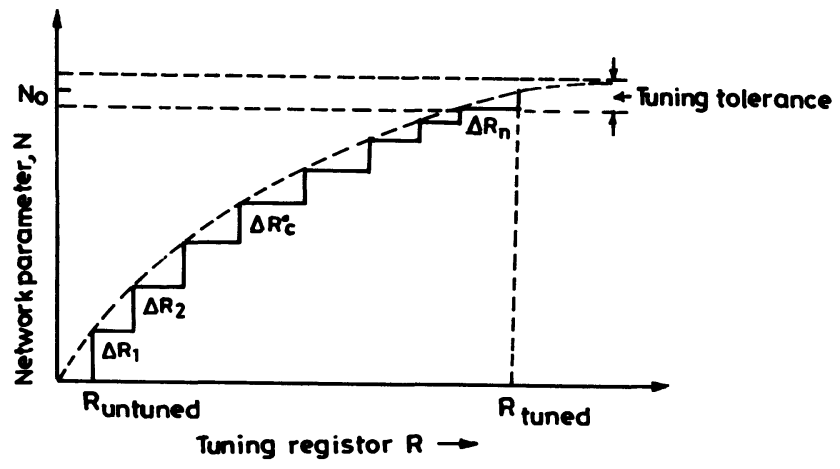

FIGURE 6

Tuning is carried out 'to values'. Hence it makes no difference whether the network is operational or not. Since the components to be tuned are generally resistors, this method consists of 'resistor trimming' in contrast to the tuning of network characteristics (e.g., amplitude, phase, frequency) that occurs in functional tuning. The method is simple (e.g., 'to-value' trimming of resistors is essential in any hybrid integrated circuits manufacturing plant) and rapid in execution (generally very few, if any, iterations are required). However, powerful computer programs are required to solve the non-linear network equations that must take firstand often second-order parasitic effects into account. Deterministic tuning is the more efficient of the two methods, but the necessary expenditure of an on-line computation facility, and the initial computational effort required, can generally be justified only by very high production volumes.

In practice, it will very often be found useful to combine functional with deterministic tuning. The initial adjustments will be carried out by deterministic tuning, where the values are obtained from either the idealized network equations or from those containing at most first-order parasitic effects. To overcome more subtle second-order parasitics, a fine-tuning step is then undertaken in which the

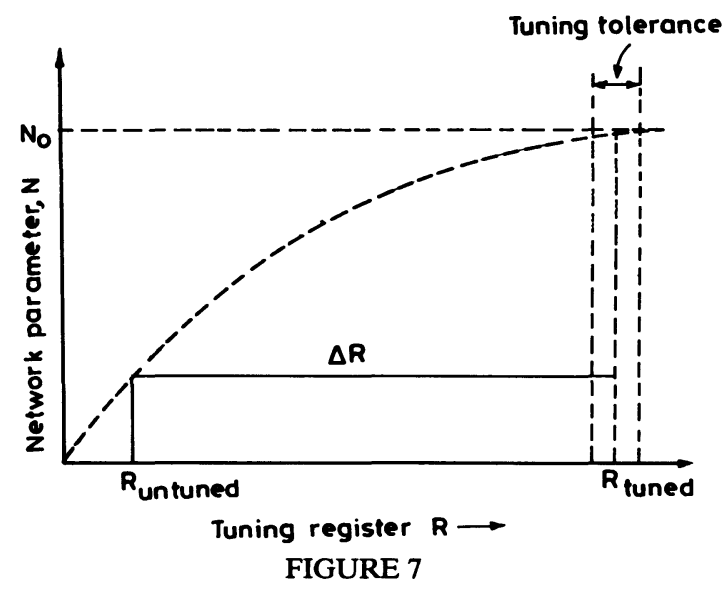


circuit is operational (i.e., assembled and powered) and a functional adjustment of one more of the critical parameters is carried out. In this way, the computational complexity inherent in deterministic-only tuning can be considerably reduced.

\section{COMPARATIVE TREATMENT OF GENERAL TUNING METHODS OF ACTIVE FILTERS}

Discussed now are the two tuning methods

(1) Functional Tuning

(2) Deterministic Tuning

FUNCTIONAL TUNING:

Functional tuning is based on a set of network equations in which the changes of the specified network characteristics $F_{j}, j=1,2, \ldots, m$, are related to incremental changes in the components $x_{i}, i=1,2, \ldots, n$, by the sensitivity matrix:

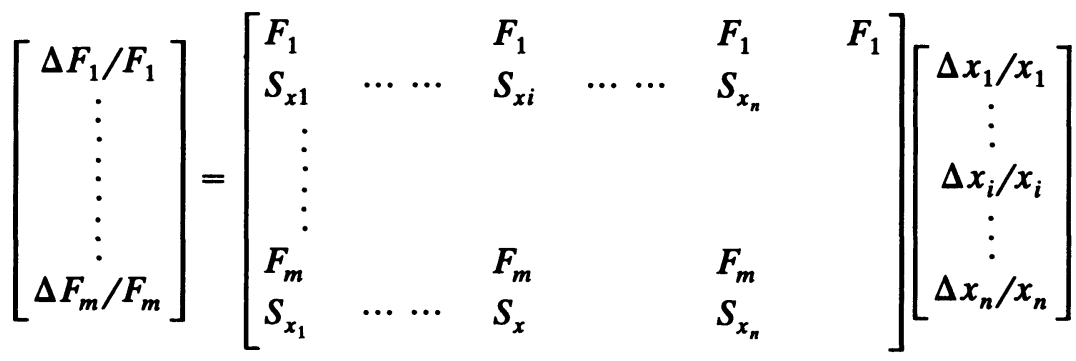

where the sensitivities $S_{x_{i}}^{F_{j}}$ are defined by

$S_{x_{1}}^{F_{j}}-\frac{d F_{j}}{d x_{i}}-\frac{x_{i}}{F_{j}}$

Hence, if $a_{i}(i=1, \ldots n)$ are the characteristic tuning parameters of a network function $F\left(a_{i}\right)$, and $R_{a j}$ are the corresponding tuning resistors (Fig. 8), then tuning the parameters from $a_{i}+\Delta a_{i}$ to $a_{i}$ will adjust the network function from its initial form $F\left(a_{i}+\Delta a_{i}\right)$ to the desired form $F\left(a_{i}\right)$. Letting:

$$
S_{R_{a j}}^{a_{i}}=\frac{d a_{i} / a_{i}}{d R_{a j} / R_{a j}}
$$




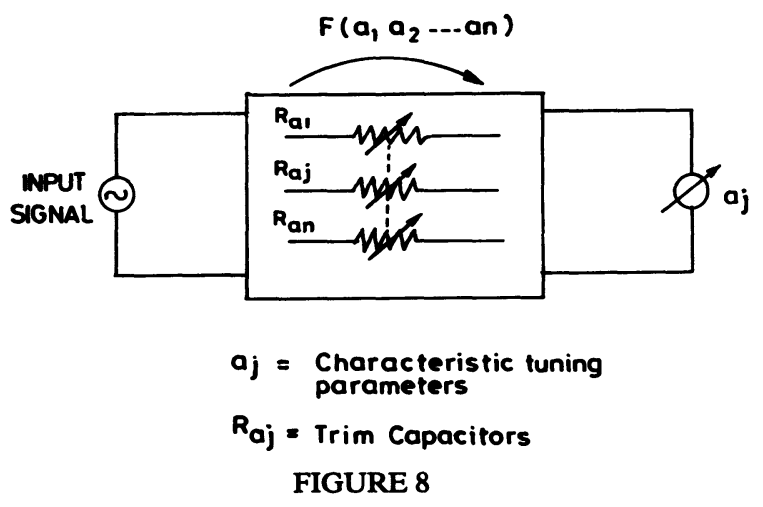

we then obtain the sensitivity relations:

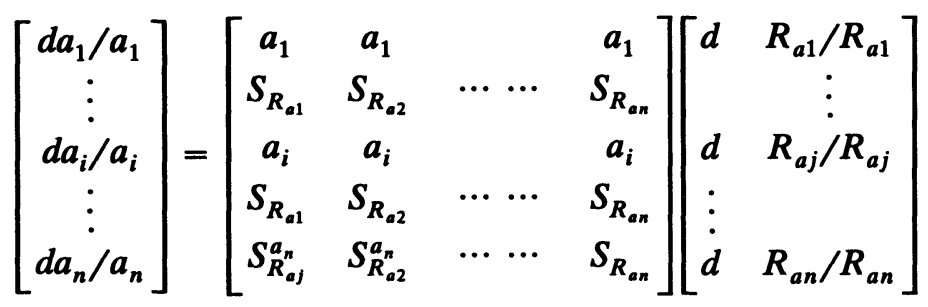

or

$\left[\frac{d a}{a}\right]=[S]\left[\frac{d R}{R}\right]$

where $[S]$ is the sensitivity matrix.

In order to allow for a non-interactive, and hence non-iterative tuning procedure, the sensitivity matrix must be a diagonal matrix, meaning that all off-diagonal matrix elements must be zero (Fig. 9(a)). In practice, this will rarely be the case. However, it may be possible to arrange the sensitivity matrix such that it is triangular with the upper triangular elements being zero (Fig. 9(b)). For each network characteristic $a_{k}$, there is then a tuning element $R_{a}$ that leaves all previously tuned parameters $a_{j}(j<k)$ unaffected. The tuning sequence is now critical; it results directly from the sensitivity matrix after the latter has been arranged in triangular form. The obtained sequence provides a single-pass (or 'one shot'), non-interactive tuning procedure requiring no iterations. If a triangular matrix in the form of Fig. 9(b) cannot be obtained accurately, it must be approximated by arranging the matrix elements such that they decrease in value to the right of the diagonal (Fig. 9(c)). In this way, the number of tuning iterations can be minimized. 


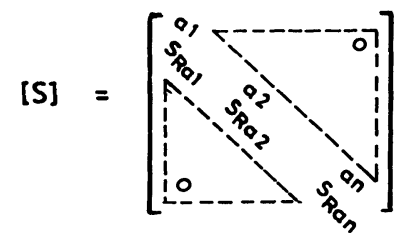

(a) Arbitrary non-iterative funing sequence

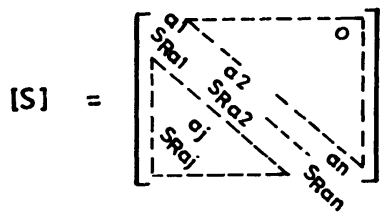

(b) Prescribed non iterative tuning sequence $R_{a_{1}} \rightarrow R_{a_{2}} \rightarrow R_{a_{j}} \rightarrow R_{a_{n}}$

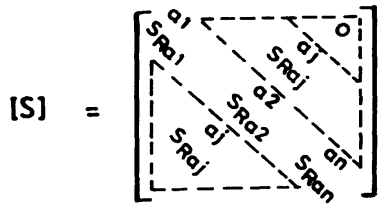

(c) Prescribed iterative timing sequence

$$
\begin{aligned}
& \mathbf{R}_{\mathbf{a}_{1}} \rightarrow \mathbf{R}_{\mathbf{a}_{2}} \rightarrow \cdots \rightarrow \mathbf{R}_{\mathbf{a}_{\mathbf{j}}} \rightarrow \mathbf{R}_{\mathbf{a}_{\mathbf{n}}} \\
& \begin{aligned}
R_{\mathbf{a}_{j}} & \rightarrow-\cdots \\
\mathbf{R}_{\mathbf{a}_{\vec{k}}} & \rightarrow \mathbf{R}_{\mathbf{a}_{\mathbf{n}}}
\end{aligned}
\end{aligned}
$$

FIGURE 9

\section{DETERMINISTIC TUNING:}

For a given network, the deterministic tuning procedure follows the flow chart presented in Fig. 10. Thus, for the second-order lowpass network shown in Fig. 11, we proceed as follows:

$T(s)=K \frac{\omega_{p}^{2}}{S^{2}+\left(\omega_{p} / Q_{p}\right) S+\omega_{p}^{2}}$,

(a) Derive the three characteristic network parameters $K, \omega_{p}$, and $Q_{p}$ as a function of the circuit components, i.e.,

$$
\begin{aligned}
K & =f_{K}\left(\beta, R_{1}, R_{2}, C_{3}, C_{4}\right) \\
\omega_{p} & =f \omega_{p}\left(R_{1}, R_{2}, R_{4}, C_{3}, C_{4}\right) \\
Q_{p} & =f_{Q p}\left(\beta, R_{1}, R_{2}, R_{4}, C_{3}, C_{4}\right)
\end{aligned}
$$

(b) Measure the capacitors $C_{3}, C_{4}$ and the closed-loop amplifier gain $\beta$. 


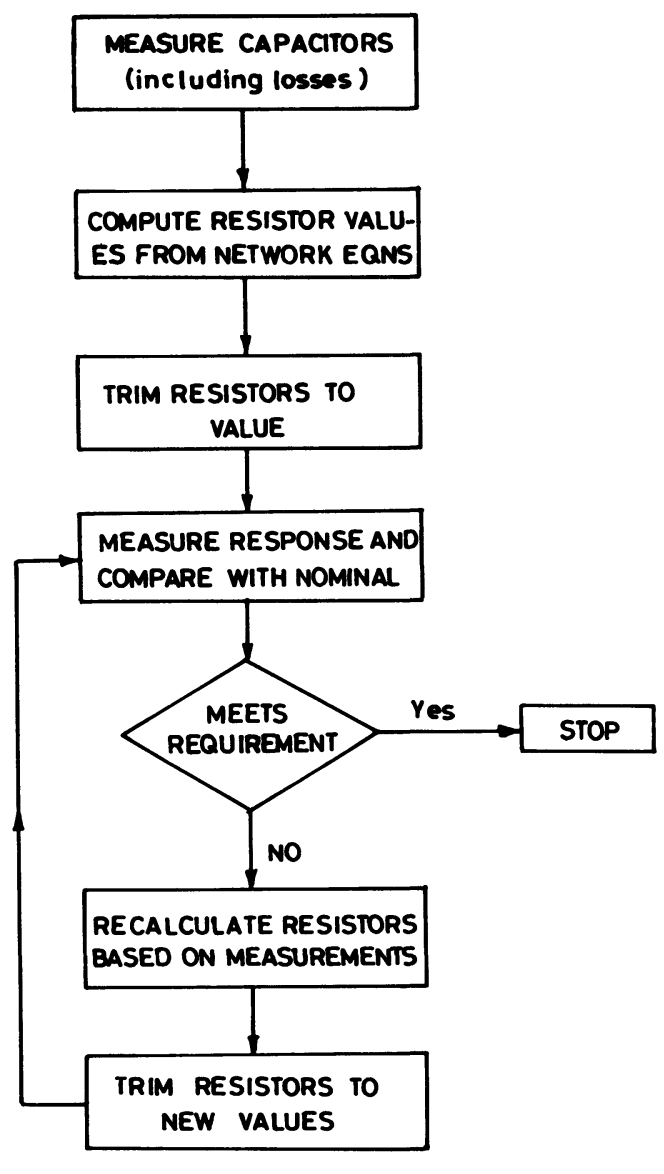

FIGURE 10

(c) Compute the following resistor values as a function of quantities that are either specified or measured, i.e.,

$$
\begin{aligned}
& R_{1}=f_{R 1}\left(K, \omega_{p}, Q_{p}, C_{3}, C_{4}, \beta\right) \\
& R_{2}=f_{R 2}\left(K, \omega_{p}, Q_{p}, C_{3}, C_{4}, \beta\right) \\
& R_{4}=f_{R 4}\left(K, \omega_{p}, Q_{p}, C_{3}, C_{4}, \beta\right)
\end{aligned}
$$

(d) Trim resistors $R_{1}, R_{2}$, and $R_{4}$ to the values computed under step (c).

In applying these four tuning steps, which are typical for the deterministic tuning procedure, the following points should be kept in mind.

(a) The equations derived in step (a) are the design equations. The derivation of the design equations precedes the tuning process and determines the nominal value of each component including the gain $\beta$. Thus, the design or 


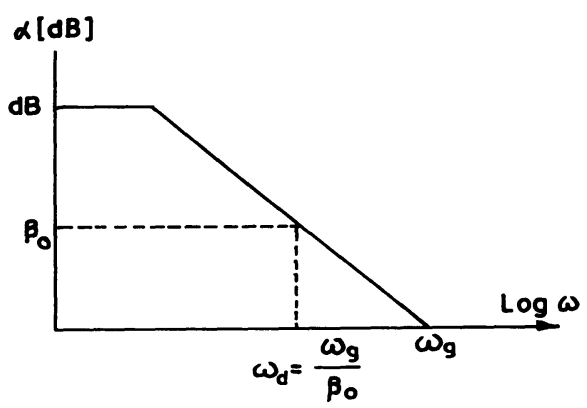

FIGURE 11

nominal value of each component is known before the tuning process is begun.

(b) The equations derived in step (c) are tuning equations and not design equations. Besides containing specified quantities (e.g., $K, \omega_{p}, Q_{p}$ ), they also contain measured quantities (e.g., $C_{3}, C_{4}$, and $\beta$ ). Thus, in the example of Fig. 11, although the nominal value of $\beta$ is known, the actual value of $\beta$ obtained in manufacture is measured in step (b) by measuring the resistors $R_{8}$ and $R_{7}$. Note that at this point the amplifier is assumed to be ideal.

(c) The circuit shown in Fig. 11 is assumed to be medium $Q\left(Q_{p} \leq 20\right)$, rather than low $Q\left(Q_{p} \leq 2\right)$, implying that tight tolerances are specified for $Q_{p}$. Thus, the closed-loop gain $\beta$ is not equal to unity, but is somewhat larger (e.g., between one and two), in order to permit a final functional correction without affecting the pole frequency $\omega_{p}$. The inclusion of the additional resistor (i.e., $R_{7}$ in Fig. 11) is a small price to pay for the increase in yield afforded by the possibility of a final touch-up tuning step. The resistor between the op amp output and inverting input terminal (e.g., $\boldsymbol{R}_{6}$ in Fig. 11) is required in any case, in order to balance d.c. effects. The deterioration of gain stability incurred by the slightly larger than unity gain is negligible, particularly in the case of hybrid-integrated filters, thanks to the very close resistor tracking obtainable with either thick or thin film resistors. The combination of deterministic and functional tuning implied here will be discussed shortly.

(d) The measurement of capacitors (i.e., $C_{3}$ and $C_{4}$ in step (b) above) should preferably be carried out after the (chip) capacitors have been assembled on the substrate. In the case of thin film capacitors there is, of course, no other choice. This permits parasitic capacitances and component drift due to circuit assembly to be taken into account during the measurement. Although methods exist for the accurate measurement of individual capacitors when they are connected to additional circuitry, the preferred way of overcoming this problem is to provide provisional capacitor contacts to the substrate edges. These permit the accurate measurement of either single capacitors, or combinations of parallel or series pairs, as required for the subsequent computations. 
To obtain a one-pass tuning procedure, parasitic effects due to non-ideal circuit components must generally be taken into account in the tuning equations derived under step (c) above. This complicates the required computations considerably. The main parasitic effects that must be contended with are:

(a) Non-ideal characteristics of the active devices (e.g., frequency dependent gain of op-amps). Referring to Fig. 12, this means that instead of using the constant gain $\beta_{0}$ in our equations, we must use $\beta(s)$; thus:

$$
\beta_{0} \rightarrow \beta(s) \simeq \frac{\omega_{g}}{S+\omega_{\alpha}}
$$

(b) Losses and frequency dependence of capacitors. Thin film capacitors for example, are both lossy and frequency dependent. If the loss of a capacitor $C_{i}$ is $\tan \delta_{i}$, then instead of $C_{i}$, we must approximate $C_{i}$ as follows

$C_{1} \rightarrow \frac{C_{i}}{1+j \tan \delta_{i}} \approx \frac{C_{i}}{1+j \delta_{i}} \approx C_{i}\left(1-j \delta_{i}-\delta_{i}^{2}\right)$

Instead of frequency-independent capacitors, thin-film capacitors are frequency dependent.

(c) Parasitic capacitances on the circuit substrate and resistive losses along conductance paths. These parasitics must be taken into account, to the extent that the response of the final assembled circuit is to be as accurate as it would be if it had been tuned functionally. In doing so, the computations required under step (c) above become rapidly more complex, the equations highly non-linear and of third, or even higher, order. With increasing complexity, only numerical solutions by computer can be expected, whereas, when assuming ideal components, analytical solutions are generally obtainable.

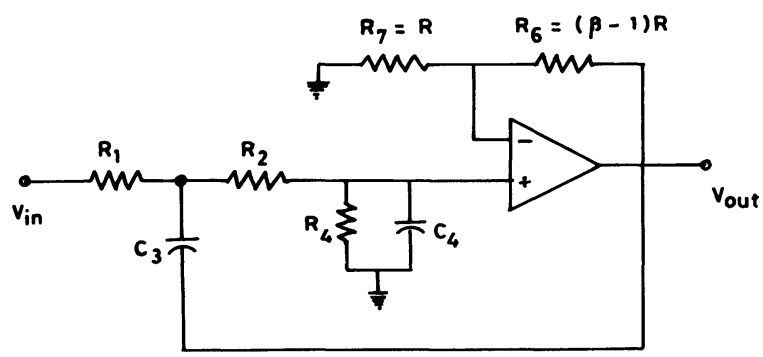

FIGURE 12 
COMBINING DETERMINISTIC WITH FUNCTIONAL TUNING:

The main characteristics of functional and deterministic tuning:

\begin{tabular}{|c|c|}
\hline Functional & Deterministic \\
\hline 1. Network is in operation & $\begin{array}{l}\text { 1. Network is not in opera- } \\
\text { tion }\end{array}$ \\
\hline $\begin{array}{l}\text { 2. Conceptually simple, directly } \\
\text { applicable }\end{array}$ & $\begin{array}{l}\text { 2. Computationally complex, re- } \\
\text { quires considerable preliminary } \\
\text { analysis. }\end{array}$ \\
\hline $\begin{array}{l}\text { 3. Trim resistors to specified } \\
\text { function values (e.g., amplitude, } \\
\text { phase, frequency) }\end{array}$ & $\begin{array}{l}\text { 3. Measure components and } \\
\text { compute resistor values, trim re- } \\
\text { sistors to computed values. }\end{array}$ \\
\hline $\begin{array}{l}\text { 4. Requires accurate phase and } \\
\text { frequency measurement }\end{array}$ & $\begin{array}{l}\text { 4. Requires accurate resistor and } \\
\text { capacitor measurements. }\end{array}$ \\
\hline $\begin{array}{l}\text { 5. Tuning steps generally inter- } \\
\text { active }\end{array}$ & $\begin{array}{l}\text { 5. Resistor trimming noninterac- } \\
\text { tive }\end{array}$ \\
\hline 6. Parasitic effects tuned out & $\begin{array}{l}\text { 6. Parasitics included in network } \\
\text { equations. }\end{array}$ \\
\hline 7. Interactive (time consuming) & 7. One to two trimming steps \\
\hline $\begin{array}{l}\text { 8. Tuning sequence derivable } \\
\text { from sensitivity matrix. }\end{array}$ & $\begin{array}{l}\text { 8. Requires on-line computation } \\
\text { and routines for the solution of } \\
\text { non-linear equations. }\end{array}$ \\
\hline $\begin{array}{l}\text { 9. Suitable for lab. purposes and } \\
\text { low-production quantities }\end{array}$ & $\begin{array}{l}\text { 10. Suitable for high-production } \\
\text { quantities. }\end{array}$ \\
\hline
\end{tabular}

Very often, the initial computational effort can be significantly reduced by deriving the equations of the idealized network and, after initial deterministic tuning, correcting the resulting error by a small number of functional tuning steps. This procedure, outlined in Fig. 13, eliminates the numerous and time-consuming iterative tuning steps generally required by the purely functional tuning procedure. It accomplishes this by reserving a small number (typically one or two) appropriate resistors for a final touch-up or vernier, functional adjustment after the circuit has previously been coarse adjusted deterministically using simple, i.e., idealized design equations.

\section{TUNING SECOND-ORDER FILTER SECTIONS:}

Normally, we would assume the kind of combined deterministic and functional tuning outlined in the previous section and presented in the flow chart of Fig. 13. The initial deterministic tuning step. would comprise the solution of the idealized 


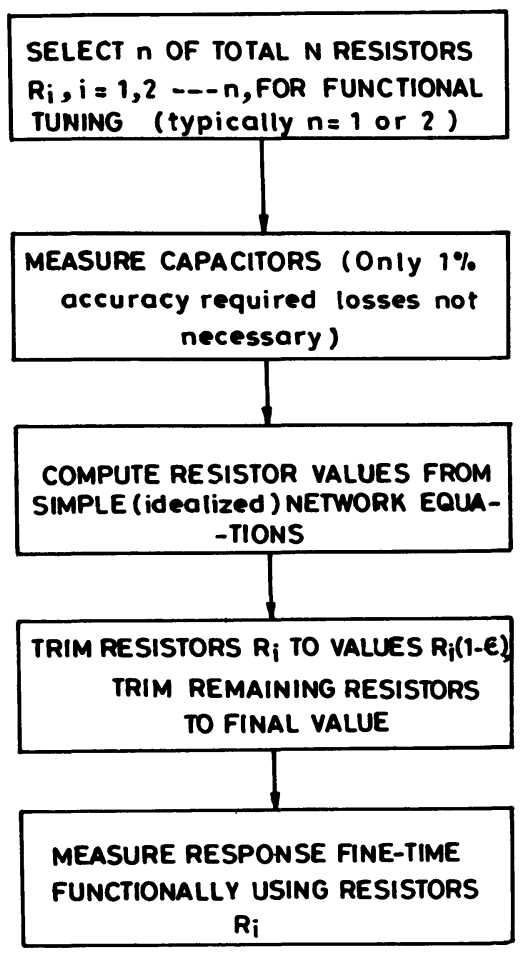

FIGURE 13

design equations while, at the same time, taking certain other constraints (e.g., minimum sensitivity, minimum gain-sensitivity product, maximum dynamic range, convenient component values) into account. The parasitic effects would not be included since they are taken into account by the subsequent functional tuning step. The component values result from a solution of the idealized design equations and the simultaneous minimization of the gain-sensitivity product. This additional constraint can be satisfied in most cases since there exist more circuit components than design equations. For the same reasons, the values of certain components (generally capacitors) can be selected from the view point of practical feasibility and minimum cost (i.e., larger values cost more). For the 2nd order filter sections, the initial computations necessary for the first deterministic tuning step are presumed to have been carried out directly in that all nominal component values are obtained from the given computer programs. The tuning resistors permit the functional step that complete the tuning procedure.

\section{A SIMPLE PHASE NETWORK FOR THE TUNING OF ACTIVE FILTERS:}

For best accuracy, second-order filter sections should be tuned 'by phase' rather than 'by amplitude'. This means that the desired pole frequency and $Q$ are tuned by adjusting appropriate 'tuning components' such that a specified phase value is 
measured at a prescribed frequency. Phase measurements and the necessity of acquiring a phase meter - typically with an accuracy of well below one degree - may sometimes be considered a drawback. In this section, we suggest a method of circumventing the need for a phase meter by providing simple, readily built auxiliary circuits with which active filters can be phase-tuned accurately enough for most applications.

Phase measurements are required primarily for the adjustment of the pole frequencies. (The notch frequencies of a band-rejection network and the right-half plane zeros of an all pass network can generally be adjusted for by using an amplitude measurement). Fortunately the phase values required for pole frequency and $Q$ adjustments are well defined and occur in multiples of $45^{\circ}$, namely $0^{\circ}, \pm 45^{\circ}, \pm 90^{\circ}, \pm 135^{\circ}$. Thus, by designing phase splitting networks that provide multiples of $45^{\circ}$ accurately and over a sufficiently wide frequency band, the pole frequencies can be adjusted without the need for a phase meter. The pole tuning is carried out with the help of the Lissajous figures on an oscilloscope.

Consider an active filter network whose phase difference between input and output is $\phi_{d}$ degrees, as shown in Fig. 14(a). We now add a phase-splitting network with the same phase shift $\phi_{d}$ to the measuring set up, as shown in Fig. 14(b). Connecting the output of the filter and the phase splitting network to the $x$ and $y$ inputs, respectively, of an oscilloscope, the resulting Lissajous figure will be a straight line with a slope of $45^{\circ}$ or $135^{\circ}$, depending on whether the phase shift between $x$ and $y$ is zero or $180^{\circ}$. Thus, to tune the active filter pole frequency and $Q$, the corresponding phase $\phi_{d}$ (which is a multiple of $45^{\circ}$ ) is obtained by tuning the corresponding tuning components until the initial ellipse degenerates to a straight line.

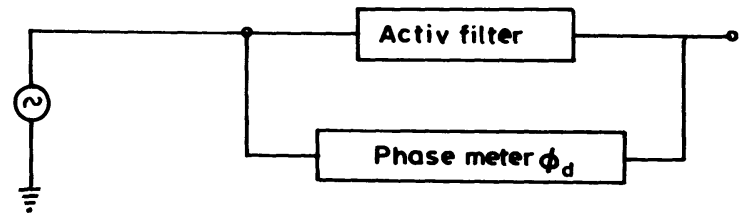

(a)

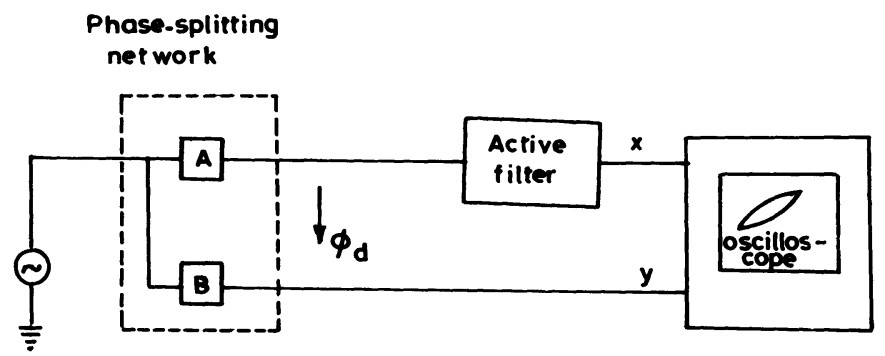

(b)

FIGURE 14 


\section{ELECTRONIC TUNING:}

An interest in electronically tunable active filters stems from the fact that there is a significant number of applications in which a small number of tunable filters may be used to replace a much larger number of non-tunable (fixed transfer function) filters. Electronic tuning has the obvious advantage, compared with mechanical tuning, that remote, automatic, and rapid adjustment of the filter transfer function is feasible. In some applications, such as automatic control, music synthesis, small range spectrum analysis, and speech synthesis, the need arises for filters with electronically controllable parameters.

Some methods of electronics tuning of active filters are presented in the next section.

\section{THEORETICAL ANALYSIS AND DESIGN PROCEDURE SECOND-ORDER TRANSFER FUNCTIONS:}

Active-filter designers usually prefer to use the output/input transfer function $T(S)$ instead of the input/output transfer function $H(S)$ used by passive-filters designers. Because active filters are usually comprised of various second-order sections, it will be helpful to study some of the possibilities.

\section{LOWPASS:}

The lowpass second-order transfer function is defined to be of the form

$$
T(S)=\frac{C}{S^{2}+\left(\omega_{P} / Q_{p}\right) S+\omega_{p}^{2}}
$$

The constant $C$ determines the level (gain or loss) of the second-order section. $\omega_{p}$ is called the un-damped natural frequency of the pole, and $Q_{p}$ is the quality of the pole. To see how these effect the transfer function $T(S)$, we can examine its magnitude.

$$
T(j w)^{2}=\frac{C^{2}}{\left(\omega^{2}-\omega_{p}^{2}\right)^{2}+\left(\omega \omega_{p} / Q_{p}\right)^{2}}
$$

The un-damped natural frequency $\omega_{p}$ helps to determine where the maximum of $T(j \omega)^{2}$ occurs. The magnitude of this maximum is determined by the quality of the pole $Q_{p}$. This is best illustrated by considering the normalized function:

$$
\frac{T(j w)}{T(0)}=\frac{\omega_{p}^{4}}{\left(\omega^{2}-\omega_{p}^{2}\right)^{2}+\left(\omega \omega_{p} / Q_{p}\right)^{2}}
$$




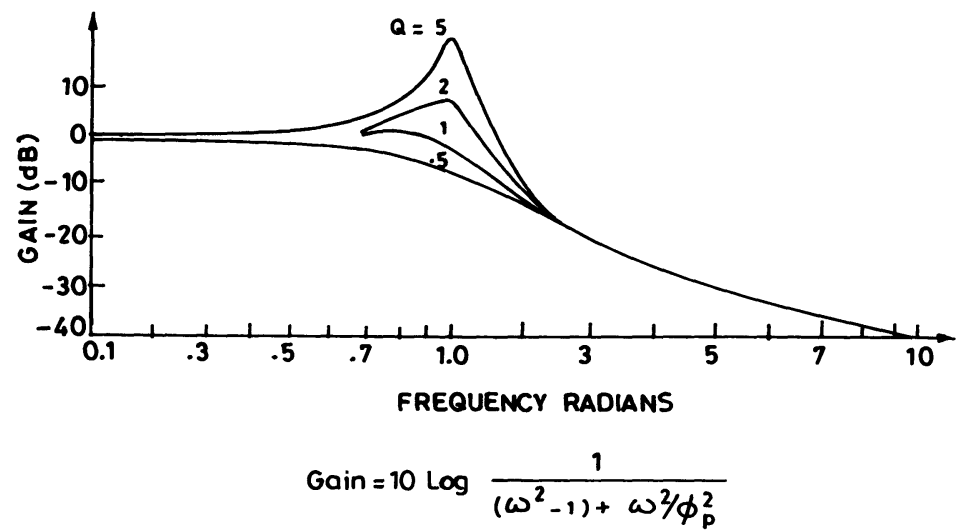

FIGURE 15

which is plotted for $\omega_{p}=1$ in Fig. 15. From the figure, it is obvious why we call it a lowpass function: it passes low frequencies (the normalized dc attenuation is $0 \mathrm{~dB}$ ) and attenuates high frequencies (at an asymptotic slope of $40 \mathrm{~dB} /$ decade).

\section{BANDPASS:}

The bandpass second-order transfer function is defined to be of the form:

$T(S)=\frac{C S}{S^{2}+\left(\omega_{p} / Q_{p}\right) S+\omega_{p}^{2}}$

The magnitude of $T(j w)$ has a maximum value at $\omega_{p}$. If we normalize with respect to this maximum value, we can write

$$
\frac{T(j \omega)}{T\left(j \omega_{p}\right)}=\frac{\left(\omega \omega_{p} / Q_{p}\right)^{2}}{\left(\omega^{2}-\omega_{p}^{2}\right)^{2}+\left(\omega \omega_{p} / Q_{p}\right)^{2}}
$$

This normalized function is plotted (for $\omega_{p}=1$ ) in Fig. 16. From the figure, it is obvious why it is called bandpass function; it passes a band of frequencies centered about $\omega_{p}$ and attenuates the low and high frequencies (with an asymptotic slope of $20 \mathrm{~dB} /$ decade)

\section{HIGHPASS:}

The highpass second-order transfer function is defined to be

$T(S)=\frac{C S^{2}}{S^{2}+\left(\omega_{p} / Q_{p}\right) S+\omega_{p}^{2}}$

This is very similar to the lowpass second-order transfer function; the low-frequency and high frequency behavior have been interchanged. Thus, for the highpass 


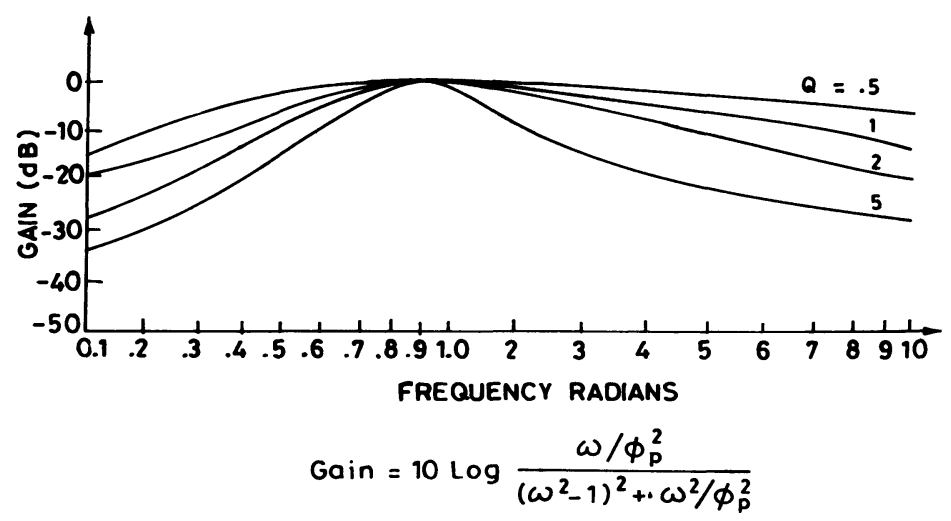

FIGURE 16

section, we normalize the magnitude response with respect to the infinite frequency response:

$$
\frac{T(j \omega)}{T(\infty)}=\frac{\omega^{4}}{\left(\omega^{2}-\omega_{p}^{2}\right)^{2}+\left(\omega \omega_{p} / Q_{p}\right)^{2}}
$$

The behavior of this function (for $\omega_{p}=1$ ) can be obtained from Fig. 15 by relabeling the abscissa to be $1 / \omega$. This indicates that the highpass function does not attenuate high frequencies but does attenuate low frequencies with an asymptotic slope of $40 \mathrm{~dB} /$ decade.

\section{STATE-VARIABLE BIQUAD:}

The representation of state-variable biquad is shown in Fig. 17. It is called biquad because it realizes an arbitrary biquadratic transfer function. A biquad active filter can be obtained by using a single-amplifier. Generally, biquad approach employs 3 op-amps per second order section. Why is the use of three op-amps in a biquad active filter when it is possible with just one? The reason is that the additional amplifiers offer us quite a few things: a very versatile network, an insensitive network, and an easily adjusted network.

The state variable biquad is shown in Fig. 17. It contains summer and two integrators as shown in Fig. 17. $a_{0}$ and $a_{1}$ shown in Fig. 17 are multipliers. It

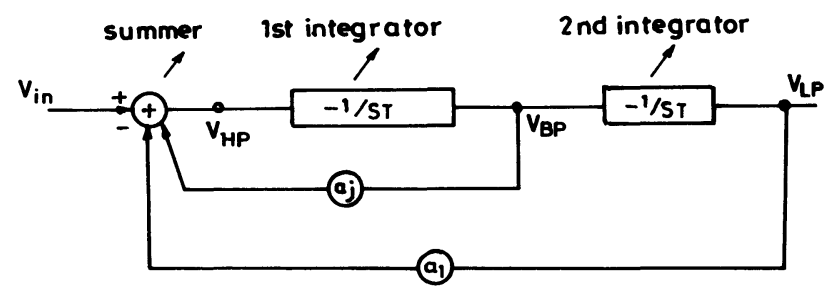

FIGURE 17 
provides the basic functions, high pass, bandpass, and lowpass simultaneously at the respective $\mathrm{O} / \mathrm{Ps}$ of the summer, the first integrator and the second integrator.

The analysis of the biquad shown in Fig. 17 is as follows:

The high pass $\mathrm{O} / \mathrm{P}$ response is given by

$V_{H P}(S)=V_{I N}(S)-a_{1} \frac{V_{H P}(S)}{S T}-a_{0} \frac{V_{H P}(s)}{S^{2} T^{2}}$

i.e. $\frac{V_{H P}(S)}{V_{I N}(S)}=\frac{1}{1+\frac{a_{1}}{S T}+\frac{a_{0}}{S^{2} T^{2}}}=\frac{S^{2}}{S^{2}+\frac{a_{1}}{T} S+\frac{a_{0}}{T^{2}}}$

Therefore, the high pass transfer function is given by

$T_{H P}(S)=\frac{V_{H P}(S)}{V_{I N}(S)}=\frac{S^{2}}{S^{2}+\frac{a_{1}}{T} S+\frac{a_{0}}{T^{2}}}$

Compare (19) with standard transfer function equation (18): Then

$$
\omega_{p}=\frac{a_{0}}{T}
$$

$\frac{\omega_{p}}{Q_{p}}=\frac{a_{1}}{T}$

Therefore $Q_{p}=\frac{a_{0}}{T} \times \frac{T}{a_{1}}=\frac{a_{0}}{a_{1}}$

The band pass transfer function is given by

$$
\begin{aligned}
& T_{B P}(S)=\frac{V_{B P}(S)}{V_{I N}(S)}=-\frac{V_{H P}(S)}{V_{I N}(S)} \times \frac{1}{S T}=-\frac{1}{S T} T_{H P}(S) \\
& \text { i.e., } T_{B P}(S)=\left(-\frac{1}{T}\right) \frac{S}{S^{2}+\frac{a_{1}}{T} S+\frac{a_{0}}{T^{2}}}
\end{aligned}
$$

The low pass transfer function is given by

$$
\begin{aligned}
& T_{L P}(S)=\frac{V_{L P}(S)}{V_{I N}(S)} \times \frac{1}{S^{2} T^{2}}=\left(\frac{1}{T^{2}}\right) \frac{1}{S^{2}+\frac{a_{1}}{T} S+\frac{a_{0}}{T^{2}}} \\
& \text { i.e. } T_{B P}(S)=\left(\frac{1}{T^{2}}\right) \frac{1}{S^{2}+\frac{a_{1}}{T} S+\frac{a_{0}}{T^{2}}}
\end{aligned}
$$




\section{THE KERWIN-HUELSMAN-NEWCOMB (KHN) BIQUAD:}

The KHN biquad is the natural choice for use as a 'Universal' filter building block out of the several existing three amplifier biquadratic filter sections based on the two-integrator loop or state variable realization of 2 nd order systems. Its salient feature is that it provides the three basic functions; high pass, bandpass, and lowpass simultaneously at the respective $\mathrm{O} / \mathrm{Ps}$ of the three amplifiers. Also, the circuit exhibits very low sensitivity with respect to the passive components and a high value of $Q$ and gain are achievable. The circuit module in this form is, in fact, available from a number of manufacturers.

The analysis of the KHN biqual is as follows:

The high pass output response is given by

$$
\begin{aligned}
V_{H P}(S)= & \left(\frac{R_{3}}{R_{1}+R_{3}}\right)\left[\left(1+\left(\frac{R_{4}}{R_{2}}\right) V_{I N}(S)+\frac{R_{1}}{\left(R_{1}+R_{3}\right)}\right.\right. \\
& \left.\left(1+\frac{R_{4}}{R_{2}}\right) V_{B P}(S)-\frac{R_{4}}{R_{2}} \times V_{L P}(S)\right] \\
= & \frac{R_{3}}{R_{1}+R_{3}}\left(1+\frac{R_{4}}{R_{2}}\right) V_{I N}(S)-\frac{1}{S R C} \times \frac{R_{1}}{\left(R_{1}+R_{3}\right)} \\
& \left(1+\frac{R_{4}}{R_{2}}\right) V_{H^{0}}(S)-\frac{1}{S^{2} R^{2} C^{2}} \times \frac{R_{4}}{R_{2}} \times V_{H P}(S)
\end{aligned}
$$

Therefore,

$$
\begin{gathered}
V_{H P}(S)\left[1+\frac{1}{S^{2} R^{2} C^{2}} \times \frac{R_{4}}{R_{2}}+\frac{1}{S R C} \times \frac{R_{1}}{\left(R_{1}+R_{3}\right)} \times\left(1+\frac{R_{4}}{R_{2}}\right)\right] \\
=\frac{R_{3}}{\left(R_{1}+R_{3}\right)}\left(1+\frac{R_{4}}{R_{2}}\right) V_{I N}(S)
\end{gathered}
$$

Therefore,

$$
\frac{V_{H P}(S)}{V_{I N}(S)}=\frac{1+R_{4} / R_{2}}{1+R_{1} / R_{3}} \times \frac{1}{1+\frac{1}{S^{2}} \frac{R_{4}}{R_{2}} \times \frac{1}{R C}+\frac{1}{S} \frac{1}{R C}} \times \frac{1+\frac{R_{4}}{R_{2}}}{1+\frac{R_{3}}{R_{1}}}
$$


The high pass transfer function is given by

$$
T_{H P}(S)=\frac{V_{H P}(S)}{V_{I N}(S)}=\frac{1+R_{4} / R_{2}}{1+R_{1} / R_{3}} \times \frac{S^{2}}{S^{2}+S \frac{1}{R C} \times \frac{.1+R_{4} / R_{2}}{1+R_{3} / R_{1}}+\frac{R_{4}}{R_{2}} \times \frac{1}{R^{2} C^{2}}}
$$

The bandpass transfer function is given by

$$
\begin{aligned}
& T_{B P}(S)=\frac{V_{B P}(S)}{V_{I N}(S)}=-\frac{1}{S R C} \times \frac{V_{H P}(S)}{V_{I N}(S)} \\
& \text { i.e., } T_{B P}(S)-\frac{1+R_{4} / R_{2}}{1+R_{1} / R_{3}} \times \frac{\left(1-\frac{1}{R C}\right) S}{S^{2}+S\left(\frac{1+R_{4} / R_{2}}{1+R_{4} / R_{1}} \times \frac{1}{R C}\right)+\frac{R_{4}}{R_{2}} \times \frac{1}{(R C)^{2}}}
\end{aligned}
$$

The low pass transfer function is given by

$$
\begin{aligned}
& T_{B P}(S)=\frac{V_{L P}(S)}{V_{I N}(S)}=-\frac{1}{S R C} \frac{V_{B P}(S)}{V_{I N}(S)}=+\frac{1}{S^{2} R^{2} C^{2}} \frac{V_{H P}(S)}{V_{I N}(S)} \\
& \text { i.e., } T_{B P}(S)-\frac{1+R_{4} / R_{2}}{1+R_{1} / R_{3}} \times \frac{\frac{1}{R^{2} C^{2}}}{S^{2}+S \frac{1}{R C} \times \frac{1+R_{4} / R_{2}}{1+R_{3} / R_{1}}+\frac{R_{4}}{R^{2}} \times \frac{1}{R^{2} C^{2}}}
\end{aligned}
$$

Thus, the design equations for the basic KHN biquad are as follows:

$$
\begin{aligned}
& T_{H P}(S)=K_{H P} \frac{S^{2}}{S^{2}+\left(\omega_{p} / Q_{p}\right) S+\omega_{p}^{2}} \\
& \text { where } K_{H P}=\frac{1+R_{4} / R_{2}}{R_{1} / R_{3}+1} \\
& T_{B P}(S)=-K_{B P} \frac{\left(\omega_{p} / Q_{p}\right) S}{S^{2}+\left(\omega_{p} / Q_{p}\right) S+\omega_{p}^{2}} \\
& \text { where } K_{B P}=\frac{R_{3}}{R_{1}}
\end{aligned}
$$




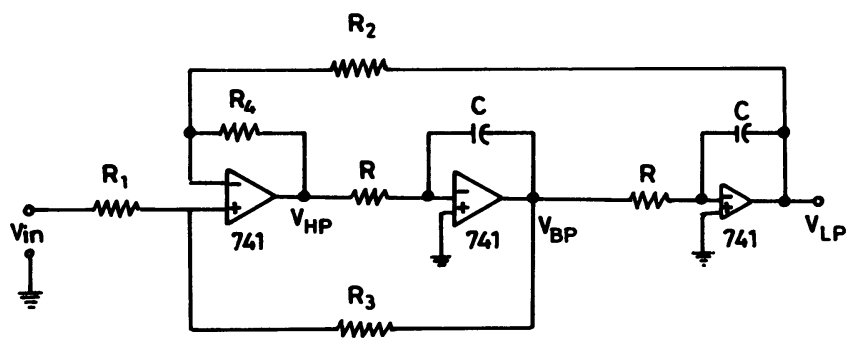

FIGURE 18

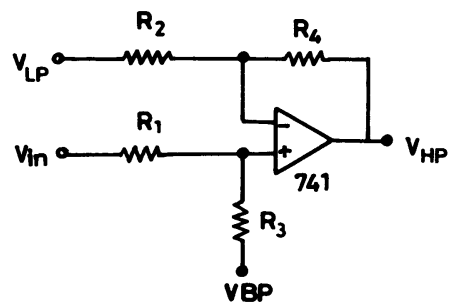

FIGURE 19

$T_{L P}(S)=K_{L P} \frac{\omega_{p}^{2}}{S^{2}+\left(\omega_{p} / Q_{p}\right) S+\omega_{p}^{2}}$

where $K_{L P}=\frac{R_{2} / R_{4}+1}{R_{1} / R_{3}+1}$

where

$$
\begin{aligned}
\omega_{p}^{2}= & \frac{R_{4}}{R_{2} \cdot R \cdot R \cdot C \cdot C .} \\
& \times \omega_{p}-\frac{R_{4}}{R_{2}} \cdot \frac{1}{R C} \\
Q_{p}= & \frac{1+R_{3} / R_{1}}{1+R_{4} / R_{2}} \frac{R_{4}}{R_{2}}
\end{aligned}
$$

\section{THE MODIFIED KHN BIQUAD:}

For electronic tunability, instead of the direct use of a FET as a voltage variable resistance to control the time constants of the integrators, the voltage-controlled variable gain amplifiers are used at the I/Ps of both the integrators. This exaggerates the effect of FET resistance variation, and a considerably larger and 


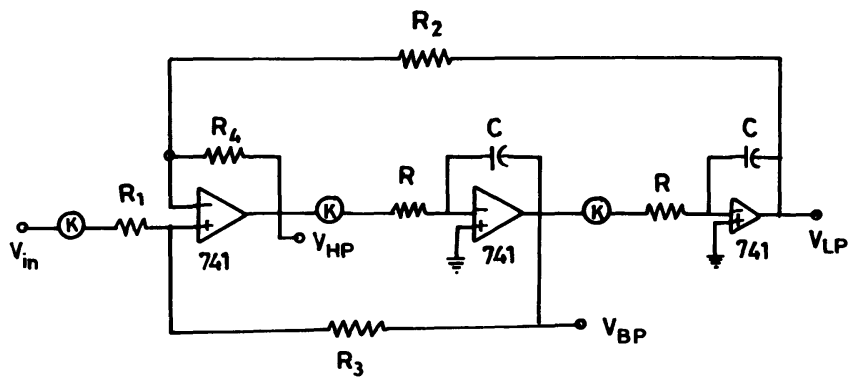

FIGURE 20

linear variation of the center frequency is achievable. The modified $\mathrm{KHN}$ is shown in Fig. 20.

Conventional tuning of this circuit involves the use of ganged resistors with manual operation. The underlying idea behind it is a variation of the time constants of the two integrators. This can however be achieved effectively through electronically varying the voltage level at the I/Os to the two integrators.

$V_{B P}(S)=\frac{-K V_{H P}(S)}{S R C}=\frac{-V_{H P}(S)}{S(R C / K)}$

where $K$ is the multiplier and $R, C$ are the passive components of the integrator. Clearly if the second multiplier ' $K$ ' were not there, we would have had

$V_{B P}(S)=-\frac{V_{H P}(S)}{S R C}$

and the only means available for varying the center frequency of the filter would be varying $R$ or $C$.

But in the present case, the time constants $R C$ has been effectively made equal to $R C / K$ by multiplying the voltage level $V_{H P}$ by a factor $K$. The action of third multiplier ' $K$ ' is similar. Theoretically, the time constant $T=R C$ of each of the two integrators has been made equal to $T / K$. Therefore, the center frequency of the filter, which is given by

$f_{0}=(2 \pi(\text { time constant of integrators }))^{-1}$

varies in the ratio $1: K$.

The use of first multiplier ' $K$ ' is to provide high pass gain as a multiple of $K$.

The voltage multiplication or gain variation for the multipliers ' $K$ ' can be simply achieved. The use of FETs as voltage controlled resistances is well known, and this can be applied to achieve voltage-controlled gain of an op-amp stage as depicted in Fig. 21. Variation of the gate voltage changes the drain-source resistance of the FET almost linearly for values of drain voltage below pinch off according to the 


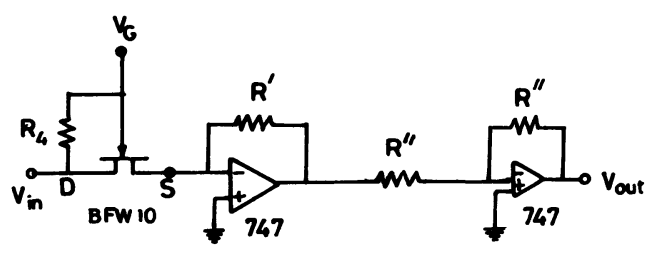

FIGURE 21

expression

$r_{d}=r_{0} \frac{\left|V_{P}\right|}{\left|V_{P}\right|-\left|V_{G}\right|}$

where $r_{0}$ is the channel resistance at zero gate voltage and $V_{p}$ is the pinch off voltage of the FET.

As a result, the gain of the op-amp stage changes correspondingly. The feedback resistance $R_{i}$ between drain and gate serves to improve and extend the linearity of operation. Analysis of Fig. 20 gives

$$
\begin{gathered}
\omega_{p}=\frac{R_{4}}{R_{2}} \cdot \frac{1}{R C} \times K \\
Q_{p}=\frac{R_{4}}{R_{2}} \cdot \frac{1+R_{3} / R_{1}}{1+R_{4} / R_{2}} \\
\omega_{p} / Q_{p}=\frac{R_{1}}{R_{3}} \frac{1+R_{4} / R_{2}}{1+R_{1} / R_{3}} \frac{K}{R C}
\end{gathered}
$$

The transfer function is given by:

$$
\begin{aligned}
T_{H P}(S) & =K \frac{1+R_{4} / R_{2}}{1+R_{1} / R_{3}} \frac{S^{2}}{S^{2}+S \frac{K}{R C} \times \frac{1+R_{4} / R_{2}}{1+R_{3} / R_{1}}+\frac{R_{4}}{R_{2}} \times \frac{1}{R^{2} C^{2}} \times K^{2}} \\
K_{H P} & =K \cdot \frac{1+R_{4} / R_{2}}{1+R_{1} / R_{3}}
\end{aligned}
$$

The bandpass transfer function is given by

$$
\begin{aligned}
T_{B P}(S) & =\frac{V_{B P}(S)}{V_{I N}(S)}=\frac{1}{S R C} \times K \cdot \frac{V_{H P}(S)}{V_{I N}(S)} \\
& =K \cdot R_{3} / R_{1} \times \frac{\left(\omega_{p} / Q_{p}\right) S}{S^{2}+\left(\omega_{p} / Q_{p}\right) \cdot S+\omega_{p}^{2}} \\
K_{B P} & =K \cdot \frac{R_{3}}{R_{1}}
\end{aligned}
$$


The lowpass transfer function is given by

$$
\begin{aligned}
& T_{L P}(S)=\frac{V_{L P}}{V_{I N}}=-\frac{1}{S R C} \times K \cdot \frac{V_{B P}}{V_{I N}} \\
& =\frac{1}{S^{2} R^{2} C_{2}} \cdot K^{2} \cdot \frac{V_{H P}}{V_{I N}} \\
& -\frac{1}{S^{2} R^{2} C^{2} / K^{2}} \times K \cdot \frac{\left(1+R_{4} / R_{2}\right)}{\left(1+R_{1} / R_{3}\right.} \\
& \frac{S^{2}}{S^{2}+S \frac{K}{R C} \times \frac{1+R_{4} / R_{2}}{1+R_{3} / R_{1}}+\frac{R_{4}}{R_{2}} \times \frac{1}{R^{2} C^{2}} \times K^{2}} \\
& =\frac{K \cdot R_{4}}{R_{2} \cdot R^{2} C^{2} / K^{2}} \cdot \frac{1+R_{2} / R_{4}}{1+R_{1} / R_{3}} \\
& \frac{1}{S^{2}+S\left(\frac{1}{R C / K} \cdot \frac{1+R_{4} / R_{2}}{1+R_{3} / R_{1}}\right)+\left(\frac{R_{4}}{R_{2}} \cdot \frac{1}{R^{2} C^{2}}\right) \times K^{2}} \\
& \text { i.e. } T_{L P}(S)=K \cdot \frac{\left(1+R_{2} / R_{4}\right)}{\left(1+R_{1} / R_{3}\right)} \cdot \frac{\omega_{p}^{2}}{S^{2}+\left(\omega_{p} / Q_{p}\right) S+\omega_{p}^{2}} \\
& K_{L P}=K \cdot \frac{\left(1+R_{2} / R_{4}\right)}{\left(1+R_{1} / R_{3}\right)}
\end{aligned}
$$

\section{TUNING ALGORITHMS FOR ACTIVE FILTERS}

The basic problem associated with the design of active filters is the sensitivity of filter characteristic to variations in filter component values. Utilization of a digital computer and the pole sensitivity concept provides a technique for filter design that incorporates sensitivity and filter tuning as an integral step in the design procedure.

Three automatic tuning methods are discussed. The discussion is broadened by a section on the computational complexity of the algorithms. Another method is discussed for the selection and ordering of tuning resistors and frequencies. This selection problem is relevant to each tuning method, although manifested differ- 
ently in each method. Lastly the tuning method used for modified KHN biquad is discussed.

\section{COMPUTERIZED DESIGN AND TUNING OF ACTIVE FILTERS:}

One of the problems associated with the design of active filters is the incorporation, as a design parameter, of the sensitivity of the filter characteristics to variations in filter components from their design values. Whenever the filter is to be manufactured on an assembly line in large quantities, components, testing, and tuning costs constitute important factors insofar as the final design configuration is concerned. In view of the number of problem variables involved, the utilization of a digital computer in the design, testing, and tuning of the filter can reduce costs, thereby increasing the probability of marketing success of the final product.

\section{MATHEMATICAL MODEL:}

Filter response characteristics are frequently specified in terms of a gain at one or more critical frequencies and perhaps a bandwidth. These design specifications are related to the values of the coefficients of the polynomials of the transfer function.

$H(S)-\frac{a_{m} S^{m}+a_{m-1} S^{m-1}+\cdots+a_{1} s+a_{0}}{S^{n}+b_{n-1} S^{n-1}+\cdots+b_{1} S+b_{0}}$

of the filter. Proper adjustment of the coefficients produces the desired response.

Variations in the values of the filter components from their design values produce corresponding variations in the coefficients of $H(S)$. These coefficient changes can be utilized to predict variations in the magnitude and phase of the filter response as a function of frequency. The classical concept of sensitivity involves the relationship between the variations in $H(S)$ and the variations in individual filter components.

The sensitivity function defined by Mason (which is the reciprocal of the original function defined by Bode) is

$S_{X_{K}}^{H(S)}=\frac{d H(S) / H(S)}{d X_{k} / X_{k}}$

Magnitude and phase sensitivities can be determined from $S_{X_{k}}^{H}$ as

$$
\begin{aligned}
S_{X_{K}}^{H(j w)} & =\frac{d|H(j w)| /|H(j w)|}{d X_{k} / X_{k}} \\
& =\operatorname{Re}\left[S_{X_{K}}^{H(S)} \mid s=j w\right]
\end{aligned}
$$

and $S_{X_{K}}^{\operatorname{Arg}[H(j w)]}=\frac{d \operatorname{Arg}[H(j w)]}{d X_{k} / X_{k}}=I_{m}\left[S_{X_{K}}^{H(S)} \mid s=j w\right]$ 
Variations in the component values also produce variations in the poles and zeros of $H(S)$, as well as the gain constant. Expressing $H(S)$ in factored form yields

$$
H(S)=\frac{G \prod_{i=1}^{m}\left(S-Z_{i}\right)}{\prod_{j=1}^{n}\left(S-p_{j}\right)}
$$

where $Z_{i}$ are the zeros, and $P_{j}$ are the poles, and $G$ is the gain constant. The zero, pole, and gain constant sensitivities are defined respectively, as

$S_{X_{k}}^{Z_{i}}=d Z_{i} /\left(d X_{k} / X_{k}\right)$

$S_{X_{k}}=d p_{j} /\left(d X_{k} / X_{k}\right)$

and

$S_{X_{k}}^{G}=(d G / G) /\left(d X_{k} / X_{k}\right)$

These root sensitivities are related to classical sensitivity by

$S_{X_{K}}^{H(S)}=\left[S_{X_{K}}^{G}-\sum_{i=1}^{m} S_{X_{K}^{i}}^{Z_{i}} /\left(S-Z_{i}\right)+\sum_{j=1}^{m} S_{X_{k}}^{p_{j}} /\left(S-p_{j}\right)\right]$

It is thus apparent that a knowledge of the pole, zero, and gain sensitivities provides the necessary information concerning composite filter sensitivity.

Utilization of root sensitivities is one practical way of dealing with simultaneous variations in a number of filter components. Practical considerations dictate that it is realistic to assume that component variations about their design values will, in general, be small enough so that only first-order effects need be considered.

$$
\begin{gathered}
\Delta G \cong \sum_{K=1}^{N}\left(\partial G / \partial X_{k}\right) \Delta X_{k} \\
\Delta Z_{i} \cong \sum_{k=1}^{N}\left(\partial Z_{i} / \partial X_{k}\right) \Delta X_{k} \\
\Delta P_{j} \cong \sum_{k=1}^{N}\left(\partial p_{j} / \partial X_{k}\right) \Delta X_{k}
\end{gathered}
$$


using matrix notation

$\left[\begin{array}{l}\Delta G \\ \Delta Z_{i} \\ \Delta P_{j}\end{array}\right]=[T]\left[\Delta X_{k}\right]$

The matrix $(T)$ can be used as the basis for determining the root sensitivities, since

$$
[S]=\left[\begin{array}{lllll}
1 / G & 1 & \cdots & \cdots & 1
\end{array}\right][T]\left[X_{k}\right]
$$

where $[S]$ is a matrix whose elements are the pole, zero, and gain sensitivities.

Since the pole, zero, and gain values for $H(S)$ are determined by the filter specifications, the design problem requires a means for computing component values to produce those desired pole, zero, and gain values.

Solving for $\Delta X_{k}$ in (56) gives

$\left[\Delta X_{k}\right]=\left[T^{-1}\right]\left[\begin{array}{l}\Delta G \\ \Delta Z_{i} \\ \Delta p_{j}\end{array}\right]$

These equations can be utilized to compute the approximate changes in the component values that are necessary to produce corrective changes in the pole, zero, and gain values.

Inversion of $[T]$ requires that it be a square non-singular matrix. The first constraint is satisfied by selecting $N=(m+n+1)$ adjustable components and the second constraint may be satisfied by a particular choice of the set $N$.

\section{DISCUSSION OF AUTOMATIC TUNING METHODS:}

In this section, three methods are presented and discussed in mutual setting. The active filter circuits of interest can be described by their transfer function $f(\omega, a, b)$, where $\omega$ is a radian frequency, $a$ is the vector of tuning resistors, and $b$ is the vector of remaining components. The general tuning problem is to find $\Delta a$ such that

$f\left(., a_{0}+\Delta a, b_{0}+\Delta b\right)-r\left(., a_{0}, b_{0}\right)$

where $a_{0}$ and $b_{0}$ are nominal values and $b$ is the undesired but measurable change in the untuned components. However, since $f$ is a rational function in $\omega$ whose coefficients are algebraic functions of $a$ and $b$, equality above is guaranteed if it holds for a particular finite number of frequencies. The methods that follow can be interpreted as techniques for solving this equation at a finite number of frequencies. 


\section{LEAST SQUARE METHOD:}

The least squares method is due to Antreich. Let the general response function.

$F: R^{\prime} \times R^{\prime} \rightarrow R^{n}$

$F(a, b)-\left[F_{1}\left(\omega_{1}, a, b\right), \ldots, F_{n}\left(\omega_{d}, a, b\right)\right]^{T}$

be composed of $n$ real valued response functions $F_{i}$ at $q$ different frequencies with $\omega, a$, and $b$ the same as before. Logical choices for the functions $F_{i}$ are the real and imaginary parts of the transfer function $N$, the magnitude and/or phase of the transfer function. Denote the Jacobian of $F$ as

$S_{F}=\left[S_{a} ; S_{b}\right]$

which is computed at the nominal values $a_{0}$ and $b_{0}$. In the tuning procedure, the response $F\left(a_{0}, b_{0}+\Delta b\right)$ is computed at the $q$ frequencies and the error vector

$\varepsilon=F\left(a_{0}, b_{0}+\Delta b\right)-F\left(a_{0}, b_{0}\right)$

is calculated. It is desired to find a connection $a$ to be made to the tuning resistors, which will minimize the error $\varepsilon$. This is formulated as

$$
\min _{x}\left\|F\left(x, b_{0}+\Delta b\right)-F\left(a_{0}, b_{0}\right)\right\|_{2}
$$

which can be identified as a non-linear least squares problem. One procedure for solving this problem is the Gauss-Newton method. The quantity within the norm is linearized and the resulting linear squares problem is solved. Additiona iterations may be used in an attempt to further reduce the error. Antreich uses a simplification of this method. Only the nominal point derivatives are used so that the solution or tuning element correction vector can be computed as

$$
\Delta a=-\left[S_{a}^{T} S_{a}\right]^{-1} S_{a}^{T} \varepsilon=-S_{a}^{+} \varepsilon
$$

Here $S_{a}^{+}$is the pseudo inverse of $S_{a}$ and can be precomputed and stored. The actual tuning procedure is to compute from (63) and then to compute the necessary adjustment from (65). If $F$ is sufficiently linear with respect to the tuning resistor vector $a$, this method should work well. Antreich used one iteration of this procedure in his example, but suggested using more if necessary. However, one should proceed cautiously since nominal point derivatives are used and the iterates may not converge. 


\section{SEQUENTIAL TUNING ALGORITHM:}

The second method is called sequential tuning and was proposed by Lopresti. Let $F$ be as before and define the quantities.

$$
\begin{aligned}
& X_{c}=\sum_{i=1}^{l}\left[\frac{\partial F}{\partial C_{i}} d C_{i}+\frac{\partial F}{\partial G_{c_{i}}} d G_{c_{i}}\right] \\
& h_{k}=G_{k} \frac{\partial F}{\partial G_{k}} \\
& u_{k}=\frac{d G_{k}}{G_{k}}
\end{aligned}
$$

Here, the $C_{i}$ 's are capacitances and the $G_{c i}$ 's are shunt conductances across the capacitors, which model dissipation effects of the capacitors. The nominal values of these conductances are usually assumed to be zero; and $G_{k}$ 's are the tuning conductances. Furthermore, all derivatives above are computed at the nominal value. Lopresti noted the problem as an optimal control problem, which was to minimize:

$I\left(u_{i}, \ldots, u_{r}, x_{i}\right)=x_{r+1}^{\nu} Q x_{r+1}+\sum_{i=1}^{r} \nu_{i} u_{i}^{2}$

Subject to

$$
\begin{aligned}
x_{k+1} & =x_{k}+h_{k} u_{k} \\
x_{1} & =x_{c}, k=1, \ldots, r
\end{aligned}
$$

where $x_{r^{\prime} 1}-d F$,the differential of $F$. The $y_{i}$ 's must be positive and are usually chosen to be diagonal in such a way to have a normalizing effect. The solution to this quadratic problem is given as

$u_{k}=H_{k} x_{k}, k=1, \ldots, r$

where the sequence of row vectors $H_{k}$ are given in enclosed form in terms of the known parameters and are computed and stored in the preproduction stage. Hence, the algorithm is to measure the components and compute $x_{C}$, and then sequentially compute the $u_{k}$ s. The best results are achieved if the resistor corresponding to $u_{i}$ is adjusted, then measured, and then the measured value is used to compute the actual achieved $u_{i}$, which is then used to compute the new $x_{i+1}$. This improvement is because there is always trimming error and this procedure uses that extra feedback beneficially. One can also choose $F$ to be the vector of transfer function coefficients. 


\section{THE LARGE-CHANGE-SENSITIVITY METHOD:}

A new large-change-sensitivity method was recently proposed by Alajajian and Trick. The foundation of the derivative is the differential form of Tellagen's theorem, which is

$\sum_{k=1}^{b}\left(\Delta V_{k} \hat{I}_{k}-\Delta I_{k} \hat{V}_{k}\right)=-\sum_{j=1}^{m}\left(\Delta V_{P_{j}} \hat{I}_{p_{j}}-\Delta I_{p_{j}} \hat{V}_{p_{j}}\right)$

The terms on the right hand side of (69) represent independent source branch voltages and currents including output post branch constraints, while the voltages and currents on the left hand side of the equation represent the remaining branch constraints. Equation (69) was derived under the assumption that there are three topologically identical networks, $N, \bar{N}$, and $N_{\Delta}$. The component values in the $N_{\Delta}$ network have been perturbed from those in $N$ such that the voltages and currents in the $N_{\Delta}$ network are $V_{k}+\Delta V_{k}$, and $I_{k}+\Delta I I_{k}$, respectively. Equation (69) relates changes in voltages and currents in the $N$ network to the voltages and currents in the $\hat{N}$ network.

Next we consider the branch constraints. The conductance branch constraints in the manufactured network $N$ are

$I_{k}=G_{k} V_{k}$

and in the tuned network $N$

$I_{k}+\Delta I_{k}=\left(G_{k}+\Delta G_{k}\right)\left(V_{k}+\Delta V_{k}\right)$

subtracting (70) from (71) gives

$I_{k}=G_{k} \Delta V_{k}+G_{k}\left(V_{k}+\Delta V_{k}\right)$

If the branch constraints of $\tilde{N}$ are chosen such that $\tilde{N}$ is the adjoint circuit of the network $N$ for example

$\hat{I}_{k}=G_{k} \hat{V}_{k}$

then the substitution of these adjoint network branch constraints and the differential branch constraints into (69) give

$\sum_{k=1}^{r}\left(V_{k}+\Delta V_{k}\right) \hat{V}_{k} \Delta G_{k}-\sum_{j=1}^{2}\left(\Delta V_{p j} \hat{I}_{p j}-\Delta I_{p j} \hat{V}_{p j}\right)$

where $r$ is the number of tuning elements and where a single input port and a single output port is assumed on the RHS of (74). Let port 1 denote the input port and port 2 denote the $O / P$ port, and assume that the $I / P$ port has a voltage source connected across its terminal pair. Since the purpose of tuning is to correct 
for derivations in response at the output port (to within a constant), the quantities of interest are the $\mathrm{O} / \mathrm{P}$ voltage $V_{0}$, or its change $\Delta V_{0}$. On this basis, choose $\widehat{V_{p 1}}=0$ and $\widehat{I_{p 2}}=1 A$ so that (74) becomes

$$
\sum_{k=1}^{r}\left(V_{k}+\Delta V_{k}\right) \hat{V}_{k} \Delta G_{k}=\Delta V_{p 2}
$$

Requiring the output voltage of the tuned filter to be within a constant of the nominal value over the frequency spectrum results in

$V_{p 2}^{j}=c V_{o d}^{j}-V_{o m}^{j}$

where $V_{o m}$ and $V_{o d}$ are the output voltages of the manufactured and nominal design circuits, respectively, the superscript $j$ denotes the frequency at which the deviation is computed, and $C$ is an unknown constant. Let $q$ represent the number of critical frequencies at which the measurements are made. Then (75) becomes

$\left[\begin{array}{ccc}\left(V_{1}+V_{1}\right) & V \ldots \ldots\left(V_{r}+V_{r}\right) V_{r} & V_{o d} \\ \vdots & & \vdots \\ \left(V_{1}+V_{1}\right) & V_{1} \ldots \ldots\left(V_{r}+V_{r}\right) V_{r} & V_{o d}\end{array}\right]\left[\begin{array}{c}\Delta G_{1} \\ \vdots \\ \Delta G_{r}\end{array}\right]-\left[\begin{array}{c}-V_{o m}^{\prime} \\ \vdots \\ -V_{o m}^{\alpha}\end{array}\right]$

In order to linearize this equation, one must take an assumption about the $\Delta V \mathrm{~s}$. If they are neglected, the consequence is that

$V_{k} \hat{V}_{k}=\frac{\partial V_{o m}}{\partial G_{k}}$

and the result is a Newton step for solving the equation

$$
\begin{aligned}
& G(y)=0 \\
& G(y)-\left[V_{o}\left(a, \omega_{1}\right) \ldots \ldots, V_{o}\left(a, \omega_{q}\right)\right]^{\nu} \\
&-C\left[V_{o d}\left(\omega_{1}\right) \ldots, V_{o d}\left(\omega_{q}\right)\right]^{\nu} \\
& y=(a, c)
\end{aligned}
$$

where $a$ is the vector of tuning conductance. The key to the large-change, sensitivity method is a different assumption about the $V$, which works much better than the Newton step.

\section{TUNING RESISTOR AND FREQUENCY SELECTION:}

A fundamental requirement common to all the algorithms is a tuning resistor and frequency selection procedure that insures good performance of the algorithm. 
The performance is directly related to this choice, and it is not immediately obvious how to make this selection when considering the interplay between the different facts involved. For many well reasoned choices of tuning resistors, the least squares and large-change-sensitivity algorithms did not converge, or the convergence was very poor in that many iterations were necessary or negative element values were generated. The resistor selection problem is more critical than the frequency selection problem. These two problems are discussed separately below.

\section{TUNING RESISTOR CHOICE:}

Since the tuning methods are attempting to solve a non-linear equation, and since there is flexibility in the choice of parameters, it is logical to choose these individual parameters, which have a strong effect on the functioning behavior. However, it is more reasonable to choose a set of parameters that, as a group, has a strong effect. This becomes clearer if one lets $F$ be the function describing the equation and $x$ the set of possible tuning parameters. For instance, let $F$ be the composite function of the magnitude of the function in (59) taken at à set of frequency points. The linearization of $F$ about $x_{0}$ is

$F(x) \cong F^{\prime}\left(x_{0}\right)\left(x-x_{0}\right)+F\left(x_{0}\right)$

Hence, the translated range space of $F^{\prime}\left(x_{0}\right)$ approximates the range space of $F$ in a small region about $x_{0}$ or in other words, the behavior of $F^{\prime}\left(x_{0}\right)$ approximates the behavior of $F$ ignoring translation. Observe that each component of $x$ corresponds to a column of $F^{\prime}\left(x_{0}\right)$ and that the number of resistors comprising $x$ will almost always be larger than the rank of $F^{\prime}(x)$ regardless of the number of component functions in $F$. This is because $F$ is composed of a rational function and typically the nature of RC active filters is such that the number of components exceeds the number of coefficients in the transfer function and obviously the maximum possible rank is the number of coefficients. Thus, one can reason that it is desirable to determine a set of 'strong' linearly independent columns of $F^{\prime}\left(x_{0}\right)$ whose span approximates the span of $F^{\prime}\left(x_{0}\right)$. These columns correspond to a set of resistors that should facilitate good movement in a region about $F\left(x_{0}\right)$.

\section{TUNING FREQUENCY CHOICE:}

There are several factors involved in the selection of tuning frequencies, and it has been found that one can heuristically choose these by keeping in mind the following discussion. The effect of the choice of a particular frequency point is that the response deviation is greatly reduced at that frequency. Because of this, and the fact that the response gradients for two closely spaced frequencies will be almost colinear, the frequencies should be reasonably spaced and placed in areas where tight control over the response is desired. The filter specifications may generate the need for tight control in certain intervals; in addition, the areas around the poles and zeros generate the need for tight control. This is because the poles and zeros determine the response and tuning frequencies placed near the 
poles and zeros have strong control over their location and, hence, strong control on the response. Furthermore, the areas near the poles and zeros are usually areas of high sensitivity; thus, the tuning components may required less adjustment. It has been found that the stopband lobes are a good place for tuning frequencies that are near the zeros; problems sometimes occurred when a tuning frequency was placed too close to zero.

\section{TUNING METHOD USED FOR MODIFIED KHN BIQUAD:}

This tuning method gives us a means of electronically controlling the function of filters. The I/P parameters are the control voltage $\left(V_{G}\right)$, the FET parameters, the element values, the op-amp parameters, and the specifications for the particular filter. The design equations have been given. The output parameters will be the gain of various filters, the center frequency, and the bandwidth. For each filter, the corresponding transfer function is obtained in the $\mathrm{O} / \mathrm{P}$. For various values of $V_{c}$, ranging from 0 to $-3.5 \mathrm{~V}$ in increments of $-0.5 \mathrm{~V}$, the output parameters and filter transfer functions are printed out.

\section{CONCLUSIONS}

A simple technique of achieving electronic tunability of active filters using controlled variable gain amplifiers has been utilized. The merits of this approach are a considerably larger and linear tuning range than hitherto achievable by known methods. It has been experimentally verified for the $\mathrm{KHN}$ biquad and found to yield excellent results. An added important advantage of electronic tunability is that we can design a digitally programmable versatile filter of this type.

Theoretically, by combining the highpass and lowpass outputs, a notch or band-reject characteristic can also be obtained from this filter. But because the KHN biquad is designed for the same cut-off frequency in both LPF and HPF, by combining the highpass and lowpass outputs, an all pass characteristic should be obtained. Because the gains $\left(K_{H P}\right.$ and $\left.K_{L P}\right)$ are the same for a particular gate voltage, we can combine both $\mathrm{HP}$ and $\mathrm{LP}$ outputs to get all pas $\mathrm{O} / \mathrm{P}$ (in this case), which will have the same gain for all frequencies. If the cut-off frequencies $\left(F_{C}\right)$ for the LPF and HPF are different and the $F_{C}$ for LPF is less than $F_{C}$ for HPF, we can obtain band-reject or notch when both LP and HP are summed.

The electronic tunability could be used at a system level to display the frequency spectrum of an incident signal. It could also be used in a mixer. If several of these filters were suitably interconnected, they could also be designed to operate as a frequency 'comb' whose frequencies could be varied electronically.

\section{REFERENCES}

1. Shao-Shiun Chung, "Practical universal active filter using generalised-imittance-convertor biquads", IEE Proc., Vol. 130, Pt. G, No. 4, August 1983, pp. 153-158.

2. L. T., Bruton, "Electronically tunable analog active filters", IEEE Trans. on Circuit Theory, May 1972, pp. 299-301. 
3. Umesh Kumar, “A decade range linearly tunable versatile filter", IEEE Trans. Inst. \& Measurements, Vol. IM-30, pp. 165-167, June 1981.

4. Henrique S. Malvar, "Electronically tunable active filters with operational transconductance amplifiers," IEEE Trans. on Circuits and Systems, Vol. CAS-29, No. 5, May 1982, pp. 333-336.

5. L. C., Thomas, "The biquad: Part-I-Some practical design considerations", IEEE Trans. Circuit Theory, Vol. CT-18, pp. 350-357, May 1971.

6. W. J., Kerwin, Huelsman, L. P., and Newcomb, R. W., "State-variable synthesis for insensitive integrated transfer functions, "IEEE J. Solid State Circuits, Vol. SC-2, pp. 87-92, Sept. 1967.

7. T. D. Shockley, and C. F. Morris, "Computerised design and tuning of active filters," IEEE Trans. Circuit Theory., Vol. CT-20, pp. 438-441, July 1973.

8. Dale E. Hocevar, and Timothy, N. Trick, "Automatic tuning algorithms for active filters," IEEE Trans. on Circuits and Systems, Vol. CAS-29, No. 7, July 1982, pp. 448-457. 

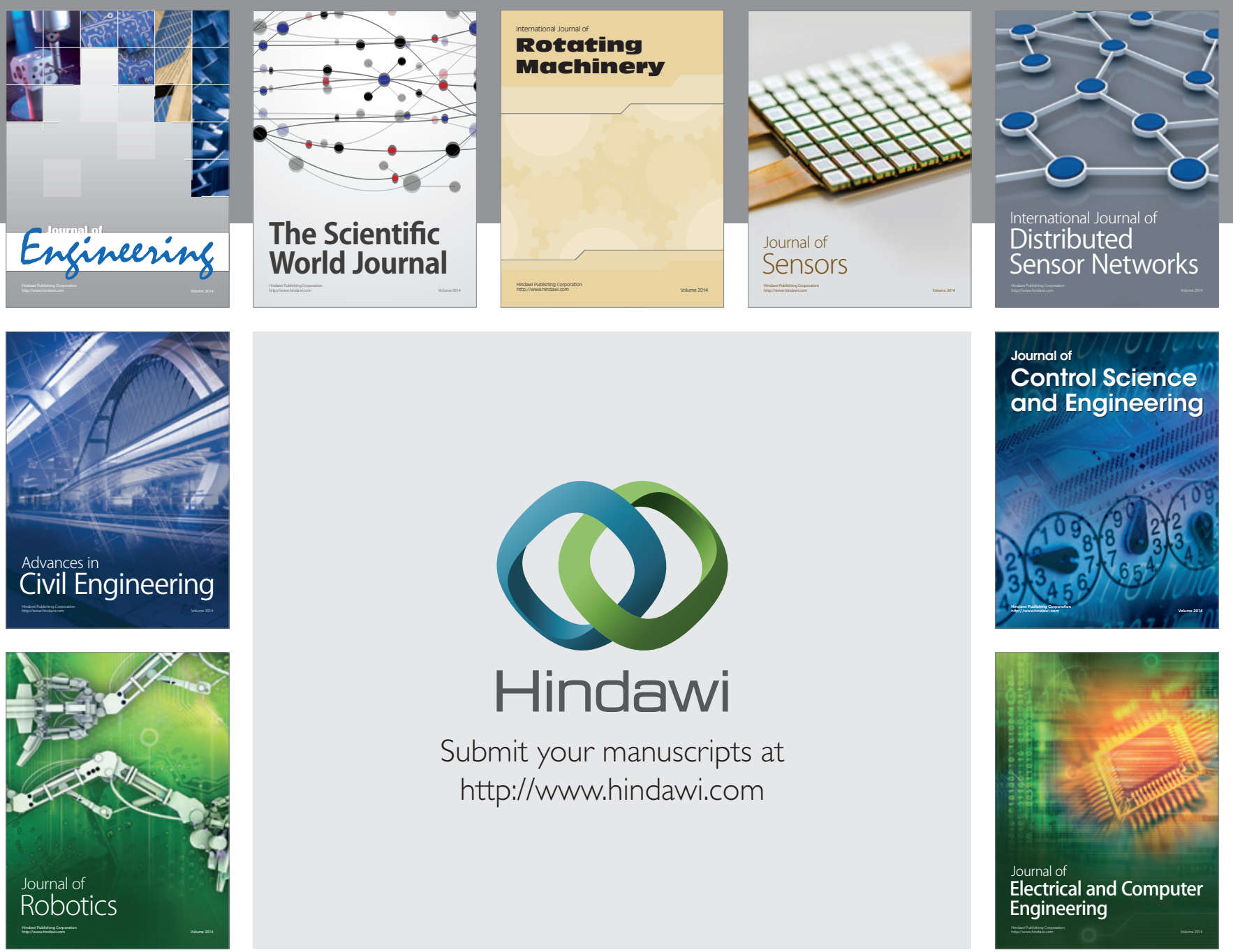

Submit your manuscripts at

http://www.hindawi.com
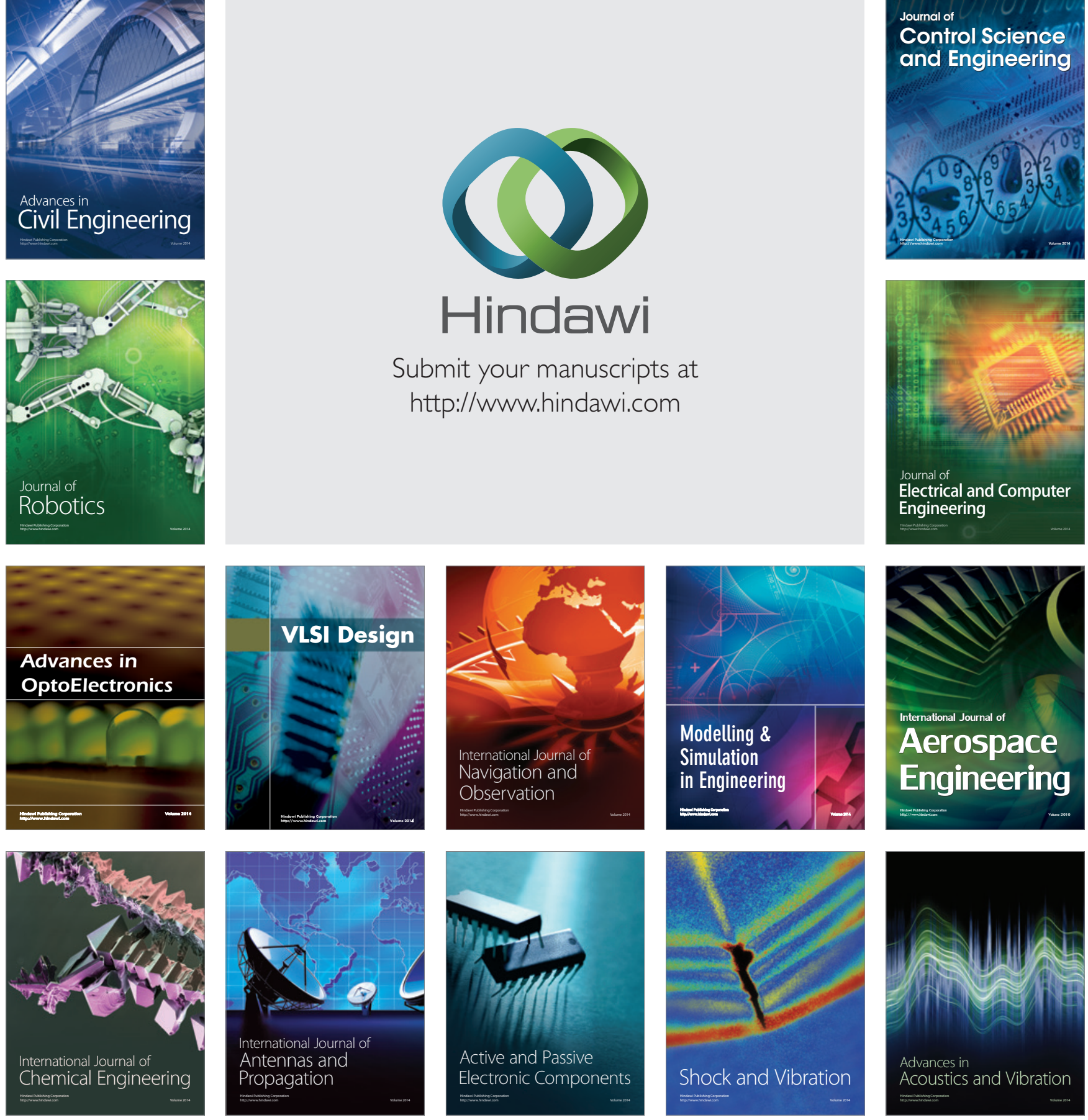